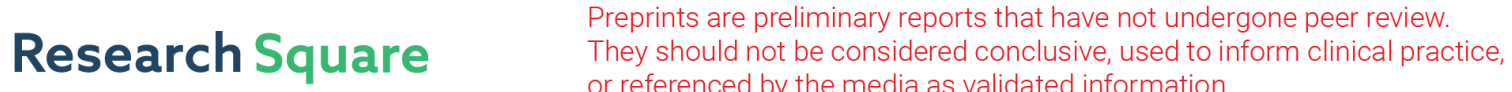 or referenced by the media as validated information. \\ Flow Estimation Only from Image Data, based on Persistent Homology
}

\author{
Tohoku University \\ Miyuki Miyazawa \\ Tohoku University \\ James Minto \\ University of Strathclyde \\ Takeshi Tsuji \\ Kyushu University \\ Ippei Obayashi \\ RIKEN \\ Yasuaki Hiraoka \\ Kyoto University \\ Takatoshi Ito \\ Tohoku University
}

Anna Suzuki ( $\square$ anna.suzuki@tohoku.ac.jp )

\section{Research Article}

Keywords: topological data analysis, persistent homology, physical properties, flow channels, fracture networks

Posted Date: March 29th, 2021

DOI: https://doi.org/10.21203/rs.3.rs-330050/v1

License: (c) (i) This work is licensed under a Creative Commons Attribution 4.0 International License.

Read Full License 


\title{
Flow Estimation Only from Image Data, based on Persistent Homology
}

\author{
Anna Suzuki ${ }^{*}$, Miyuki Miyazawa ${ }^{1}$, James Minto², Takeshi Tsuji ${ }^{3,4}$, Ippei Obayashi ${ }^{5}$, \\ Yasuaki Hiraoka ${ }^{6}$, and Takatoshi Ito ${ }^{1}$ \\ ${ }^{1}$ Institute of Fluid Science, Tohoku University, Sendai 980-8577, Japan \\ ${ }^{2}$ Department of Civil \& Environmental Engineering, University of Strathclyde, Glasgow, UK \\ ${ }^{3}$ Department of Earth Resources Engineering, Kyushu University, Fukuoka 819-0385, Japan \\ ${ }^{4}$ International Institute for Carbon Neutral Energy Research (WPI-I2CNER), Kyushu University, \\ Fukuoka 819-0385, Japan \\ ${ }^{5}$ Center for Advanced Intelligence Project, RIKEN, Tokyo 103-0027, Japan \\ ${ }^{6}$ Kyoto University Institute for Advanced Study, ASHBi, Kyoto University, Kyoto 606-8501, Japan
}

*corresponding author(s): Anna Suzuki (anna.suzuki@tohoku.ac.jp)

\begin{abstract}
(147 words)
Topological data analysis is an emerging concept of data analysis for characterizing shapes. A state-of-the-art tool in topological data analysis is persistent homology, which is expected to summarize quantified topological and geometric features. Although persistent homology is useful for revealing the topological and geometric information, it is difficult to interpret the parameters of persistent homology themselves and difficult to directly relate the parameters to physical properties. In this study, we focus on connectivity and apertures of flow channels detected from persistent homology analysis. We propose a method to estimate permeability in fracture networks from parameters of persistent homology. Synthetic 3D fracture network patterns and their direct flow simulations are used for the validation. The results suggest that the persistent homology can estimate fluid flow in fracture network based on the image data. This method can easily derive the flow phenomena based on the information of the structure.
\end{abstract}

\section{Introduction}

Fluid flow processes are ubiquitous in the world, and most are governed by the geometry and nature of the surrounding structures. In particular, recent miniaturization of artificial devices has led to the need for understanding and controlling flow in finer structures. It is also attracting attention to understand flow behaviors in complex fracture networks in developments of natural resources, as in the case of shale gas and geothermal developments.

It has been a long-term scientific challenge to predict flow behavior of porous media from structural properties. Permeability is a key parameter for examining flow phenomena in porous media ${ }^{1}$. Permeability cannot be determined only from structure data, and needs to be obtained from laboratory experiments or numerical fluid flow simulations. In contrast, porosity is a parameter that is often used to characterize the structures. The porosity-permeability correlation has been studied extensively in the literature to estimate permeability using porosity (so-called Kozeny-Carman equation) ${ }^{2,3}$. This Kozeny-Carman equation provides a relationship between structure and flow. The correlation has been studied extensively in the literature to estimate permeability using porosity. However, no matter how much void there are, if they are not connected, water cannot flow. Therefore, the Kozeny-Carman equation does not always work. The correlation has been modified to represent real phenomena by adding parameters such as fractal dimension, and tortuosity ${ }^{2}$. These additional parameters can only be determined by fitting, which is not the best way to go about flow prediction based on structural information.

Let us also consider flow in a channel from an inlet to an outlet. Hagen-Poiseuille equation is a physical law that describe a steady laminar flow of a viscous, incompressible, and Newtonian fluid through a circular tube of constant radius, $r$. This is an exact solution for the 
flow, can be derived from the (Navier-) Stokes equations, and is another way of expressing the relationship between structure and flow. Using Darcy's law, a representative permeability, $K_{\mathrm{HP}}$ $\left[\mathrm{m}^{2}\right]$, for the capillary can be calculated depending only on the radius:

$$
K_{H P}=\frac{r^{2}}{8}
$$

Similarly, for flow in a fracture bounded by two smooth, parallel walls, the permeability, $K_{\mathrm{CL}}$ $\left[\mathrm{m}^{2}\right]$, can be calculated depending only on the aperture, $h[\mathrm{~m}]$ :

$$
K_{C L}=\frac{h^{2}}{12}
$$

Since the flow rate is proportional to the cube of the fracture aperture, this relationship between flow and aperture is well-known as the "cubic law" 4-7.

Eqs. (1) and (2) are only ways to obtain simplified analytic solution to describe the relationship between the flow and structures. This is another way to predict permeability from structural properties ${ }^{8}$. In natural rocks, it is not always a single fracture, but multiple fractures that form a network. Thus, it is necessary to understand not just an individual fracture, but how channels are connected from an inlet to an outlet in whole networks. There have been many studies focusing on networks, but most of the parameters used to describe the structure are probabilistic variables that capture individual fractures, and no suitable parameters have been found yet to evaluate the flow of the entire networks 9 .

Topology, a branch of modern mathematics, is good at roughly investigating the connectivity of shapes. Topology focuses on the properties (called topological properties or topological invariants) that are preserved when some form (shape or space) is continuously deformed (stretched or bent, but not cut or pasted). Topology can extract global features that are difficult to capture with machine learning and convolutional neural networks, so it is promising as a complementary feature to extract image information that cannot be detected with other methods. It can be applied to volumetric data as well, so it can pick up information that has been missed in one-way slice-by-slice analysis common to many forms of data processing.

Several studies used topological invariants to describe pore-scale structures in porous materials and fracture networks ${ }^{10,11}$. The Minkowski functionals can be interpreted as area, perimeter, or the Euler characteristic, which is a topological constant and were used to link to hydraulic properties ${ }^{12,13}$. Scholz et al. (2012) ${ }^{14}$ showed an empirical expression of permeability with the Minkowski functionals Liu et al. (2017) ${ }^{15}$ showed the correlation of relative permeability to one of the topological invariants called Euler characteristic. Armstrong et al. $(2019)^{16}$ reviewed the theoretical basis of the Minkowski functionals and its application to characterize porous media. Counting the number of holes using topological invariants like they did is a clue to the shape of the object, and the "essential information" can be extracted well. On the other hand, topology too narrowly focuses on the essential information, it also discards a lot of information, such as size of pore space. The size information, such as radii of tube or apertures of fractures in Eqs. (1) and (2), must be detected to determine permeability derived analytically. Therefore, previous studies had to add the size information in other ways.

Homology is a standard technique for identifying a topological space. In particular, the concept of homology has traditionally played a role in feature extraction focusing on the existence of "holes". Here, the "hole" structure can be regarded as a connected flow channels from an inlet to an outlet. It is expected that topology can be used to detect such connected flow channels. 
By tracking the sequence of topological spaces, namely, by recording how long homological features persist, we can add information about the size and length of the holes. This can give us a quantitative indication of the size of the holes and the amount of space available, which is called persistent homology. Persistent homology is one of the most important tools in topological data analysis and is expected to compute geometric and topological features of various shapes with ease of computation ${ }^{17-20}$. Thus, this has been applied in several research fields ${ }^{21-25}$, and is also beginning to be used in the analysis of porous materials ${ }^{26-31}$.

At this point, in contrast to topological invariants, persistent homology can provide a lot of information that we might need, but it is difficult to interpret the parameters of persistent homology themselves ${ }^{27-30}$. Ushizima et al. $(2012)^{26}$ estimates permeability of porous rocks by using Reeb graphs to represent the pore networks. They use persistent homology to distinguish between significant and "noisy" pore spaces, and to supplement the Reeb graphs. Their paper did not go into quantitative evaluation, but focused on qualitative evaluation and visualization. As mentioned before, the "hole" structure that is characterized by topology, can be regarded as connected flow channels from an inlet to an outlet. The aim of our study is to detect the flow channels by persistent homology. Suzuki et al. $(2020)^{31}$ proposes a method to detect flow channels in 2D images from persistent homology through image processing. By using their image processing procedure, persistent homology is expected to detect such connected flow channels in complex fracture networks and would also provide their size information such as apertures to predict the permeability.

In this study, we applied persistent homology to estimate permeability in fracture networks based on image data. Persistent homology was used to detect the number of flow channels and their apertures in the networks. Synthetic fracture networks were generated, and direct flow simulation was conducted. Permeability derived from persistent homology and simulation results were compared. We applied the proposed method to several published image data and discussed the applicability of permeability estimation based on persistent homology.

\section{Results \\ Detection of flow channels from persistent homology analysis}

An example of a fractured rock model with a flow channel connecting an inlet to an outlet is shown in Figure 1a. The yellow area is a solid skeleton, while the white area is fractures forming void spaces. The connecting fractures from the top (inlet) to the bottom (outlet) can be a flow channel. In persistent homology analysis, such structure is recognized as "hole" and quantified as a 1-dimensional hole. Additionally, a discrete island (i.e., connected component) is quantified as a 0 -dimensional hole, and a ball (i.e., enclosed solid voids) is quantified as a 2-dimensional hole. The numbers of $k$-dimensional holes (the dimension of the $k$ th homology vector space) are known to the $k$ th Betti number $\left(\beta_{0}, \beta_{1}\right.$, and $\left.\beta_{2}\right)$. This study focuses on "hole" structures penetrating from an inlet to an outlet, which can be flow channels hence we only analyze 1-dimensional holes in this study.

One aspect of persistent homology analysis is that independent fractures are recognized as 2-dimensional holes, as shown in Figure $1 \mathrm{~b}$. These independent fractures would not contribute to the fluid flow. Therefore, we can distinguish fractures that act as flow channels and independent fractures by 1-dimensional holes and 2-dimensional holes. 


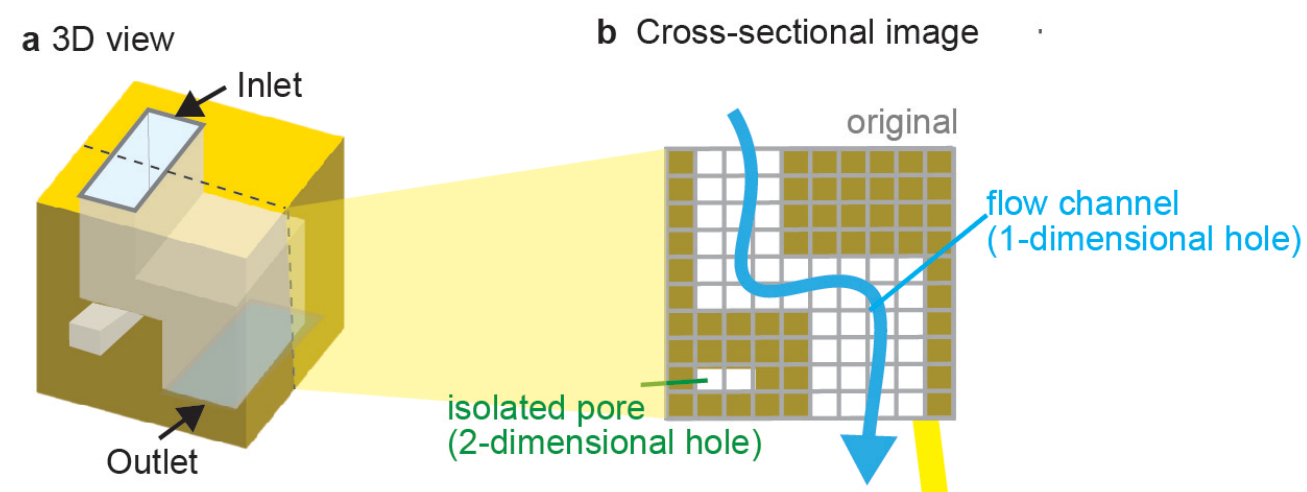

c Filtration

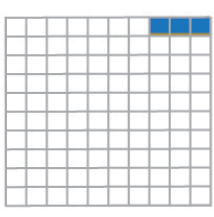

$t=-5$

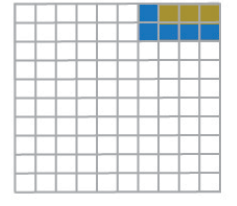

$t=-4$

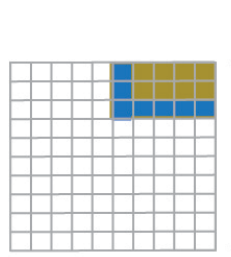

$t=-3$

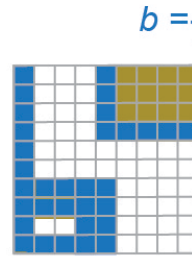

$t=-2$
$15 \mathrm{~mm}$

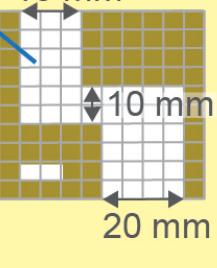

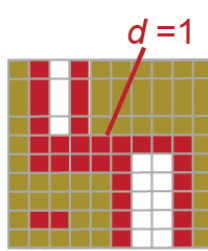

$t=1$

time

145

Figure 1 | Persistent homology analysis for fracture networks by HomCloud ${ }^{32}$. a 3D view and $\mathbf{b}$ cross-sectional image of fracture network with a flow channel (light blue arrow) and an isolated pore (mentioned as green). c Schematic of filtration process. Image at $t=-1$ is the original image. White grids express void spaces. Blue grids are the grids that were removed during the thinning process. Red grids are the grids that were added during the thickening process.

Much research has explored various applications of persistent homology in statistical data analysis of point cloud data ${ }^{22,33,34}$. Since the purpose of this study is to analyze the information of structures based on the image data (e.g., micro-CT images), binarized digital images were used for analysis. Jiang et al. (2018) ${ }^{29}$ applied persistent homology to analyzed rock pore geometries obtained from micro-CT images. The rock pore geometries were first represented as sphere cloud data using a pore-network extraction method, then analyzed by calculating the Vietoris-Rips complex topology of the input sphere cloud data. We used an open software HomCloud (https://homcloud.dev/) to analyze binarized 3D images, which can obtain the information of persistent homology by calculating the Euclidian distance of $2 \mathrm{D}$ or 3D black and white images ${ }^{32}$.

Figure 1c shows an example of data process in our persistent homology analysis, called filtration ${ }^{17}$. In filtration, the solid skeletons (yellow parts) are made thinner or thicker, voxel-by-voxel. The original image is set to -1 . The process of thinning yellow voxels adjacent to white voxels is regarded as -1 , while the process of thickening yellow voxels adjacent to white voxels is regarded as +1 . When we reduce the time, the space eventually becomes empty. The nested sequence of the topological spaces from the empty space to the filled space is recorded. The times when the hole appears or disappears are called "birth time" or "death time", expressed as " $b$ ". or " $d$ ", respectively.

In filtration (Figure 1c), a point of the flow channel is closed at $t=1$. This closed point is the narrowest aperture in the flow channel. Taking advantage of this, the length of narrowest aperture can be obtained as death time $d$ multiplied by two and its resolution $\delta$. (narrowest 
174 aperture $=2 \times d \times \delta=2 \times 1 \times 5=10$ ). It has been known that the narrowest width in flow 175 channels, which is called critical pore radius, correlates with permeability better than other pore 176 radii ${ }^{35-37}$. Detecting narrowest aperture by persistent homology can therefore be useful to 177 estimate the permeability.

178 The set of pairs $\left(b_{i}, d_{i}\right)$ for $k$-dimensional holes is called $k$ th persistence diagram, $\mathrm{PD}_{k}$. 179 If pairs of negative $b$ and positive $d(b<0<d)$ are detected in $\mathrm{PD}_{1}$, the pairs suggest "hole" 180 structures (i.e., flow channel) presenting in the original image. If there are multiple hole 181 structures, multiple birth-death pairs are obtained in the $b<0<d$ domain of $\mathrm{PD}_{1}$. Each value 182 of $d$ indicates each narrowest aperture of multiple fracture channels. The ability to link between 183 the numbers of flow channels and its narrowest apertures is one of the strengths of persistent 184 homology analysis.

185 Here is something to keep in mind. A 1-dimensional hole detected by persistent 186 homology is a flow channel penetrating from an inlet to an outlet. At the same time, a ring187 shaped, internal void-structures is also detected as a 1-dimensional hole. Figure $2 \mathrm{a}$ shows an 188 example of a ring-shaped internal void structure. This structure does not connect to the outside 189 (i.e., no flow channel). However, during filtration, the internal void space is closed at $t=1(d$ $190=1$ ). If images include such hole structures, it would overestimate the number of flow channels. 191 Now, let us prepare an inverted image that the yellow and white are reversed as shown in Figure 192 2b. In filtration, a ring appears at $t-1$, and the ring width is detected by the value of $b(b=-1)$. 193 Therefore, we can expect to detect only the hole fractures that act as flow channels by 194 subtracting the holes recognized in the inverted image from the holes recognized in the original 195 image. We set the number of 1-dimensional holes (i.e., Betti number) obtained from the 196 original and inverted figures to $\beta_{1}$ and $\bar{\beta}_{1}$, respectively. The number of flow channels can be derived as $\beta_{1}-\overline{\beta_{1}}=1-1=0$ as shown in Figure $2 \mathrm{a}$ and $2 \mathrm{~b}$.

Another example is shown in Figure 2c. This is a ring-shaped internal void structure with two channels that are connected to the outside. In this case, there are two 1-dimensional holes $\left(\beta_{1}=2\right)$ with $d=1$ and $d=2$. Figure $2 \mathrm{~d}$ is the inverted image of Figure $2 \mathrm{c}$. There are a 1-dimensional holes $\left(\beta_{1}=1\right)$ with $d=1$. By subtracting the holes recognized in the inverted image from the holes recognized in the original image, the number of flow channels can be calculated as $\beta_{1}-\overline{\beta_{1}}=2-1=1$. At the same time, persistent homology analysis provides the narrowest aperture of the flow channel by $d=2$. From these analyses, we estimate the number of channels and their narrowest aperture by using the inverted image in this study. 


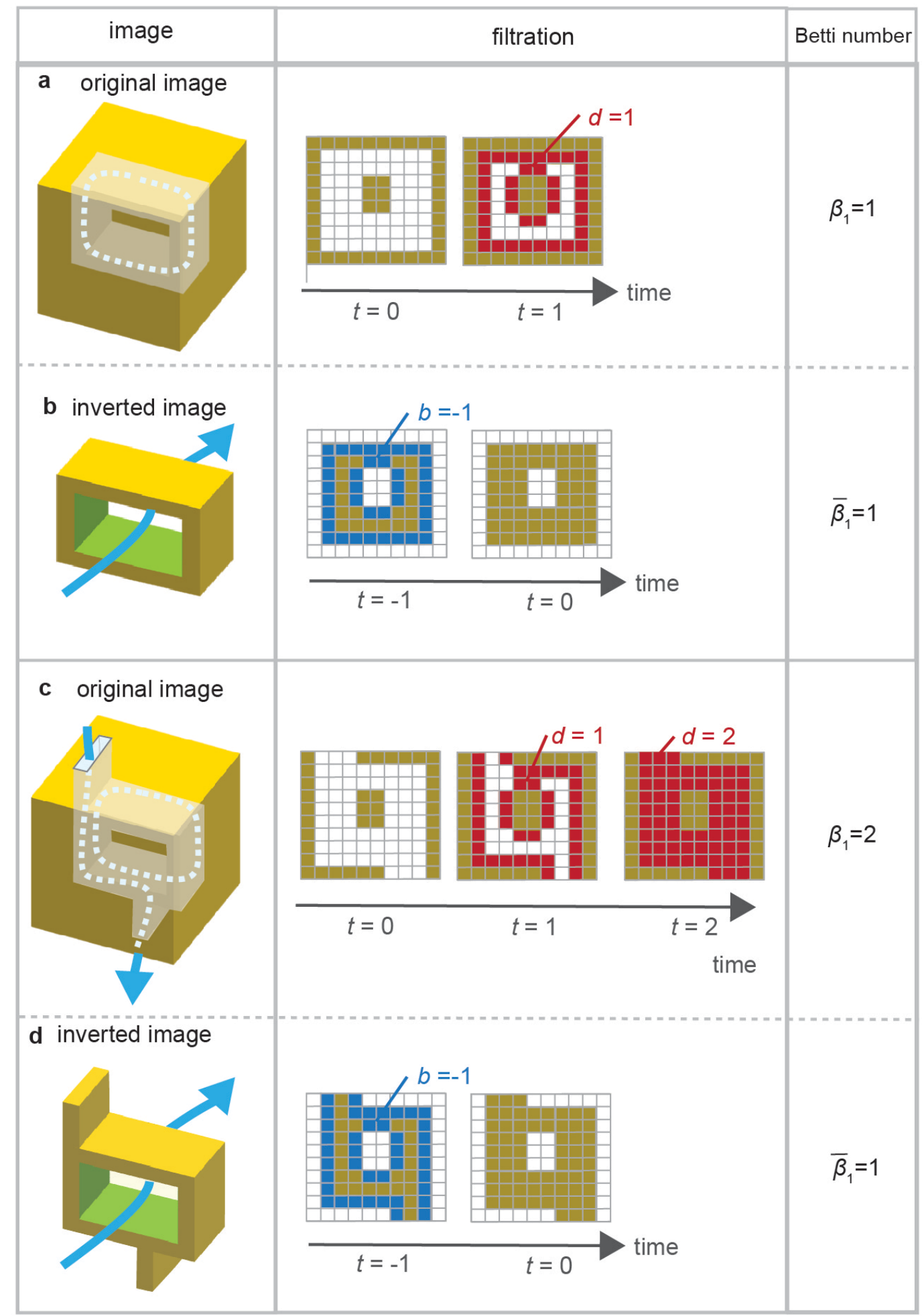

208 Figure 2 | Detecting flow channels using inverted images a ring-shaped, internal 209 void-structure that is not connected to the outside, and $\mathbf{b}$ its inverted image. $\mathbf{c}$ ring210 shaped, internal void-structure with two channels that is connected to the outside and 211 forms a flow channel. $\mathbf{d}$ its inverted image. The left column shows 3D view of images. 212 The center column describes processes of filtration. The right column lists Betti 213 numbers $\beta_{1}$. 


\section{Synthetic fracture network}

Synthetic fracture networks were generated by using OpenSCAD

217 (https://www.openscad.org/). We distributed multiple penny-shaped fractures by controlling 218 the apertures, radii, numbers, and orientations of fractures to generate a fracture network ${ }^{38}$. By 219 hollowing out the generated fracture network from a rectangular block, a fractured model where the void spaces were composed of the fracture network was created. This study characterizes one-dimensional flow. The top surface was an inlet, and the bottom surface was an outlet. The fractures were connected from the top to the bottom surfaces. The side boundaries were closed, and water did not flow out from the side.

The fractured model is shown in Figure 3. Figure $3 \mathrm{a}$ and $3 \mathrm{~b}$ are the outside and the

a

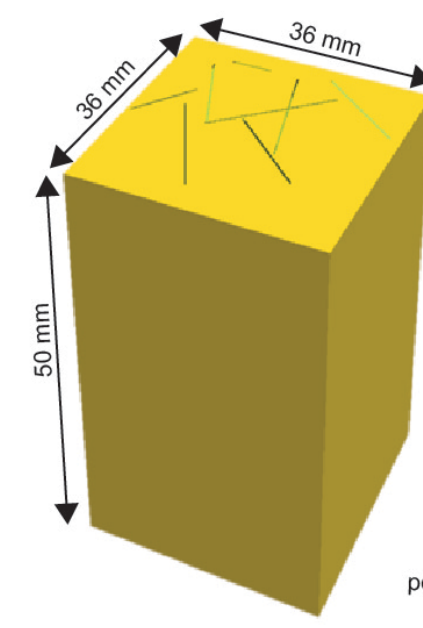

c Orthogonal distribution

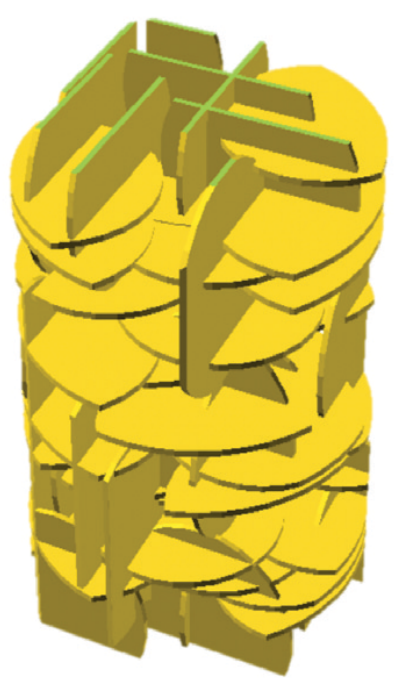

b

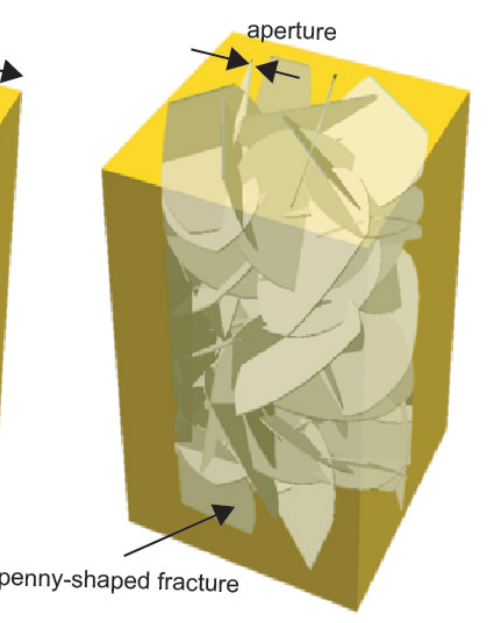

d Random distribution

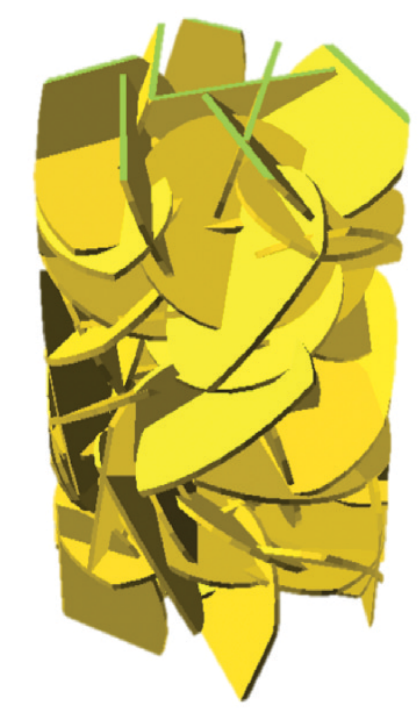

Figure 3 | Fractured models. a outside and $\mathbf{b}$ inside of model. c Orthogonal distribution and $\mathbf{d}$ random distribution of fracture networks. 
Table 1 | Fracture network parameters and results of permeability.

236

237

238

239

240

241

242

243

244

\begin{tabular}{|c|c|c|c|c|c|c|}
\hline & \multicolumn{4}{|c|}{ Fracture network parameters } & \multirow[b]{2}{*}{$\begin{array}{l}\text { Simulation } \\
\text { result }\end{array}$} & \multirow[b]{2}{*}{$\begin{array}{c}\mathrm{PH} \\
\text { estimation }\end{array}$} \\
\hline Model & $\begin{array}{l}\text { Diameter } \\
\quad(\mathrm{mm})\end{array}$ & $\begin{array}{c}\text { Aperture } \\
(\mathrm{mm})\end{array}$ & $\begin{array}{c}\text { Fracture } \\
\text { density } \\
\text { parameter }\end{array}$ & $\begin{array}{c}\text { Number } \\
\text { of } \\
\text { fractures }\end{array}$ & & \\
\hline \multicolumn{7}{|c|}{ Orthogonal } \\
\hline 01 & 10 & 0.2 & 3000 & 77 & $1.04 \times 10^{-10}$ & $2.47 \times 10^{-10}$ \\
\hline $\mathrm{O} 2$ & 10 & 0.6 & 3000 & 77 & $3.03 \times 10^{-9}$ & $3.43 \times 10^{-9}$ \\
\hline $\mathrm{O} 3$ & 10 & 1.0 & 3000 & 77 & $1.31 \times 10^{-8}$ & $1.55 \times 10^{-8}$ \\
\hline $\mathrm{O} 4$ & 5 & $0.2-1$ & 6100 & 234 & $1.58 \times 10^{-10}$ & $2.68 \times 10^{-10}$ \\
\hline O5 & 10 & $0.2-1$ & 1720 & 66 & $4.00 \times 10^{-10}$ & $7.18 \times 10^{-10}$ \\
\hline O6 & 5 & $0.2-1$ & 2020 & 251 & $3.50 \times 10^{-10}$ & $2.70 \times 10^{-10}$ \\
\hline $\mathrm{O} 7$ & 5 & $0.2-1$ & 13000 & 230 & $1.12 \times 10^{-10}$ & $2.16 \times 10^{-10}$ \\
\hline 08 & $5-25$ & $0.2-1$ & 895 & 73 & $3.15 \times 10^{-9}$ & $2.20 \times 10^{-9}$ \\
\hline \multicolumn{7}{|l|}{ Random } \\
\hline R1 & 10 & 0.2 & 2000 & 77 & $1.01 \times 10^{-10}$ & $2.36 \times 10^{-10}$ \\
\hline $\mathrm{R} 2$ & 10 & 0.6 & 2000 & 77 & $2.90 \times 10^{-9}$ & $3.46 \times 10^{-9}$ \\
\hline R3 & 10 & 1.0 & 2000 & 77 & $1.28 \times 10^{-8}$ & $1.39 \times 10^{-8}$ \\
\hline R4 & 10 & 0.2 & 1000 & 38 & $3.42 \times 10^{-11}$ & $1.12 \times 10^{-10}$ \\
\hline R5 & 10 & 0.2 & 3000 & 115 & $1.75 \times 10^{-10}$ & $3.70 \times 10^{-10}$ \\
\hline R6 & 25 & 1.0 & 280 & 11 & $5.21 \times 10^{-9}$ & $4.03 \times 10^{-9}$ \\
\hline $\mathrm{R} 7$ & 5 & 0.2 & 11950 & 459 & $1.30 \times 10^{-10}$ & $1.36 \times 10^{-10}$ \\
\hline
\end{tabular}

\section{Estimation of fracture numbers and apertures by persistent homology}

$3 \mathrm{D}$ image data of each fractured model $(36 \mathrm{~mm} \times 36 \mathrm{~mm} \times 50 \mathrm{~mm}$ with a voxel resolution $\delta$ of $0.1 \mathrm{~mm}$ ) were binarized and analyzed by persistent homology using HomCloud $^{32}$. The image size was $360 \times 360 \times 500$ voxels.

The estimated narrowest fracture apertures based on the persistent homology analysis are shown in Figure 4. Fracture networks (O1-O3, R1-R3) distributes a single value of fracture aperture of $0.2 \mathrm{~mm}, 0.6 \mathrm{~mm}$, and $1.0 \mathrm{~mm}$, respectively. Figures $4 \mathrm{a}$ and Figure $4 \mathrm{~b}$ shows the results for the orthogonal and the random fracture networks, respectively. As mentioned before, the narrowest apertures in each flow channel were calculated as $2 d_{i} \delta$ in persistent homology analysis. The values given in each network $(0.2 \mathrm{~mm}, 0.6 \mathrm{~mm}, 1.0 \mathrm{~mm})$ are compared with the estimated narrowest apertures $\left(2 d_{i} \delta\right)$. The sizes of the circles represent the number of birthdeath pairs with $d_{i}$. As shown in Figure 4, the estimated narrowest apertures are equal or relatively larger than the actual values given in the network. Most of results are between one or two times larger than the actual values. Persistent homology analysis detects not fractures themselves but flow channels.
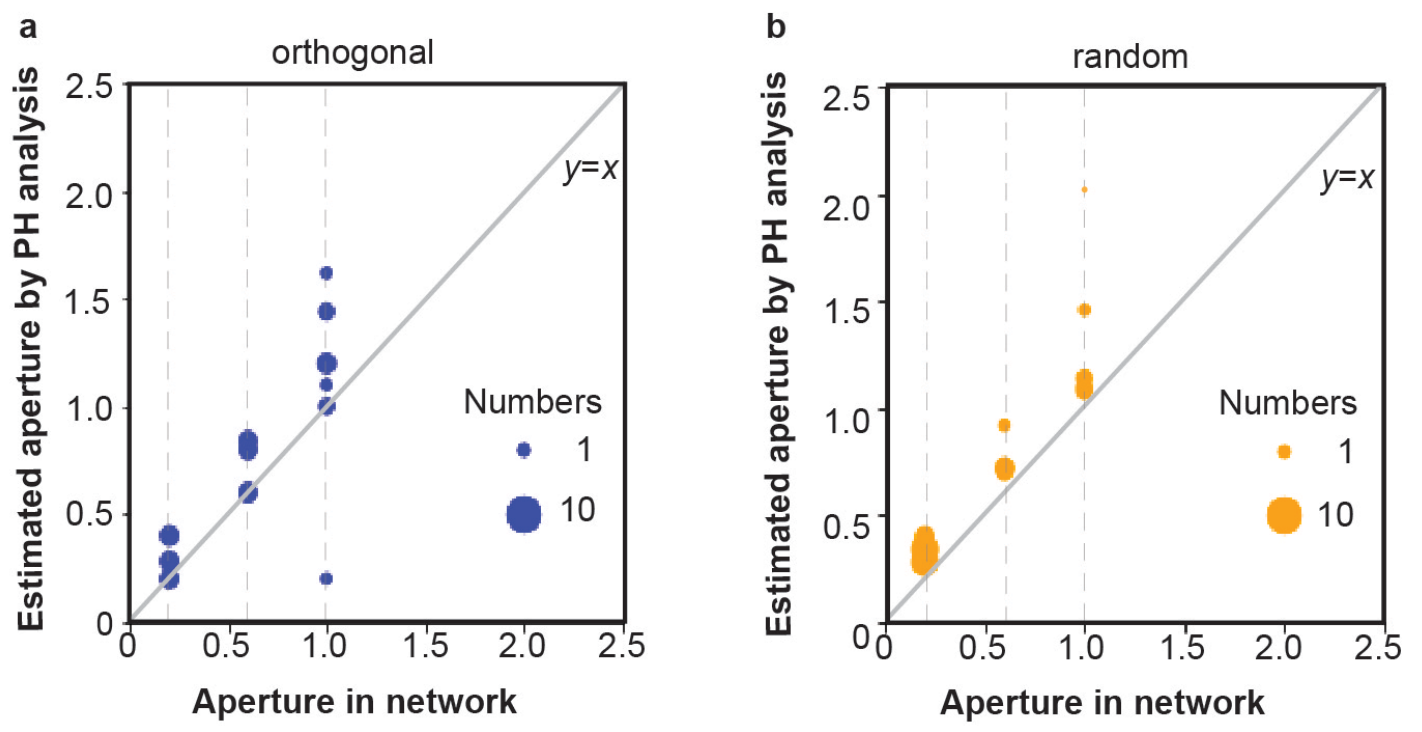


\section{Derivation of permeability}

We use Eq. (2) to derive permeability, which can be originally calculated by comparing the Stokes equation with the Darcy's law. If Assuming that a fracture is a smooth and parallel plate with the aperture of $h$ and that there is a uniform pressure gradient in one direction within the plane of the fracture, the total volumetric flowrate in the fracture can be written as

$$
Q_{x}=-\frac{w h^{3}}{12 \mu} \frac{d P}{d x}
$$

263

264

265

266

267

where $w$ is the width of the fracture, perpendicular to the flow direction. $h$ is the aperture, and $\mu$ is the water viscosity, $d P / d x$ is the pressure gradient. Darcy's law describes one-dimensional fluid flow through porous media as

$$
Q_{x}=-\frac{K A}{\mu} \frac{d P}{d x}
$$

where $A$ is the cross-sectional area. Comparison of Eqs. (3) and (4) shows that the permeability of the fracture can be identified as

$$
K=-\frac{w h^{3}}{12 A}
$$

If the cross-sectional areas of the inlet and outlet are assumed to be wh, Eq. (5) becomes Eq. (2). If we consider the case of parallel multiple channels, the permeability can be derived in the following equation

$$
K=\sum_{i=1}^{N} \frac{w_{i} h_{i}^{3}}{12 A}
$$

where $A$ is the surface area of the cross section of the medium, and $N$ is the number of flow channels. $w_{i}$ is the depth of flow channel, and $h_{i}$ is the aperture of the flow channel $i, i=1, . ., N$. There is an unknown parameter $w_{i}$ in Eq. (6). The $3 \mathrm{D}$ voxel data can be regarded as a series of $2 \mathrm{D}$ cross-sectional images. The $2 \mathrm{D}$ cross-sectional image data provides total area of pore space, $A_{p}$ in each layer. If we introduce effective depth $\bar{w}$ that is the same for all flow channels, $\bar{w}$ can be derived by $\bar{w}=\frac{\min \left(A_{p}\right)}{\sum_{i=1}^{N} h_{i}}$ where $\min \left(A_{p}\right)$ is the minimum of total area of pore space for all layers. The number of flow channels $N$ was estimated from the number of birth-death pairs, and the aperture $h_{i}$ was estimated as $2 d_{i} \delta$ in persistent homology analysis. Thus, Eq. (6) can be written as follows

$$
K=\frac{\bar{w}}{12 A} \sum_{i=1}^{N}\left(2 d_{i} \delta\right)^{3}
$$

\section{Estimation of permeability from persistent homology analysis}

Before applying complex fracture networks, we validated Eq. (7) and our simulation with simple fracture models. Simple models with one or two fractures penetrating from an inlet to an outlet were used (see Figure 5a). Apertures and number of fractures in each model are listed in Table 2. Direct flow simulation with the same fracture network was conducted in OpenFOAM (https://www.openfoam.com/). We could obtain volumetric flow rate and pressure gradient between the inlet and the outlet to calculate equivalent permeability based on Darcy's law. Comparison of permeability between flow simulation and persistent homology analysis is shown in Figure 5b, and listed in Table 2. The persistent homology estimation is in 
very good agreement with the simulation results. The results show that for such a simple system, persistent homology can estimate the permeability well using Eq. (7).

Table 2 I Estimation for simple parallel model.

\begin{tabular}{c|c|c|c|c|c:c}
\hline Model & $\begin{array}{c}\text { Aperture } \\
\text { (mm) }\end{array}$ & $\begin{array}{c}\text { Number of } \\
\text { fractures }\end{array}$ & $\mathbf{d}$ & $\boldsymbol{\beta}_{\mathbf{1}}-\overline{\boldsymbol{\beta}_{\mathbf{1}}}$ & $\begin{array}{c}\text { Simulation } \\
\text { result }\end{array}$ & $\begin{array}{c}\text { Estimation from } \\
\text { PH analysis }\end{array}$ \\
\hline S1 & 1.0 & 1 & 10 & 1 & $1.80 \times 10^{-9}$ & $1.80 \times 10^{-9}$ \\
S2 & 1.0 and 1.0 & 2 & 10 and 10 & 2 & $3.66 \times 10^{-9}$ & $3.47 \times 10^{-9}$ \\
S3 & 0.5 & 1 & 5 & 1 & $2.39 \times 10^{-10}$ & $2.17 \times 10^{-10}$ \\
S4 & 0.5 and 0.5 & 2 & 5 and 5 & 2 & $4.79 \times 10^{-10}$ & $4.33 \times 10^{-10}$ \\
S5 & 0.1 & 1 & 1 & 1 & $1.95 \times 10^{-12}$ & $1.73 \times 10^{-12}$ \\
S6 & 0.1 and 0.1 & 2 & 1 and 1 & 2 & $3.89 \times 10^{-12}$ & $3.47 \times 10^{-12}$ \\
S7 & 0.5 and 1.0 & 2 & 5 and 10 & 2 & $2.00 \times 10^{-9}$ & $1.95 \times 10^{-9}$ \\
\hline
\end{tabular}

a

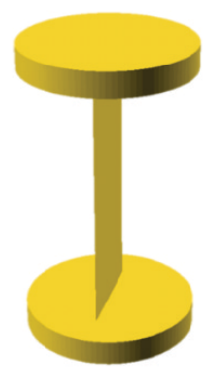

306

307

308

309

310 b
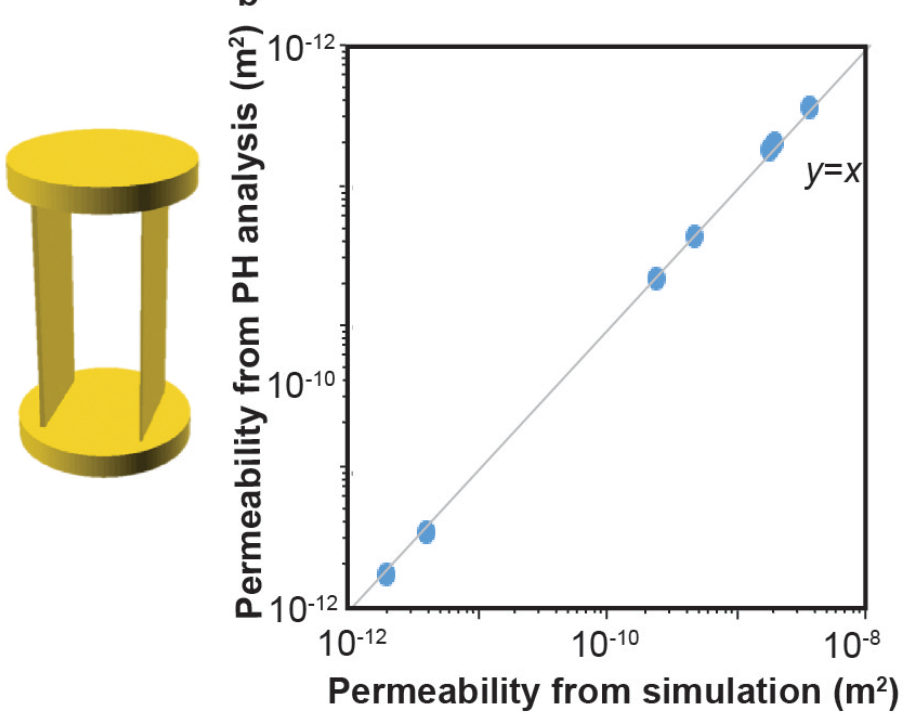

Figure 5 | Validation with simple models. a one or two fracture penetrating the model. The top and bottom are the inlet and the outlet. $\mathbf{b}$ Comparison of permeability between persistent homology $(\mathrm{PH})$ analysis and direct simulation. The calculated permeability is listed in Table 2.

Next, we applied Eq. (7) to complex fracture networks listed in Table 1. Comparison of permeability between flow simulation and persistent homology analysis is shown in Figure 6 . The estimation is in reasonable agreement with the simulation results. 


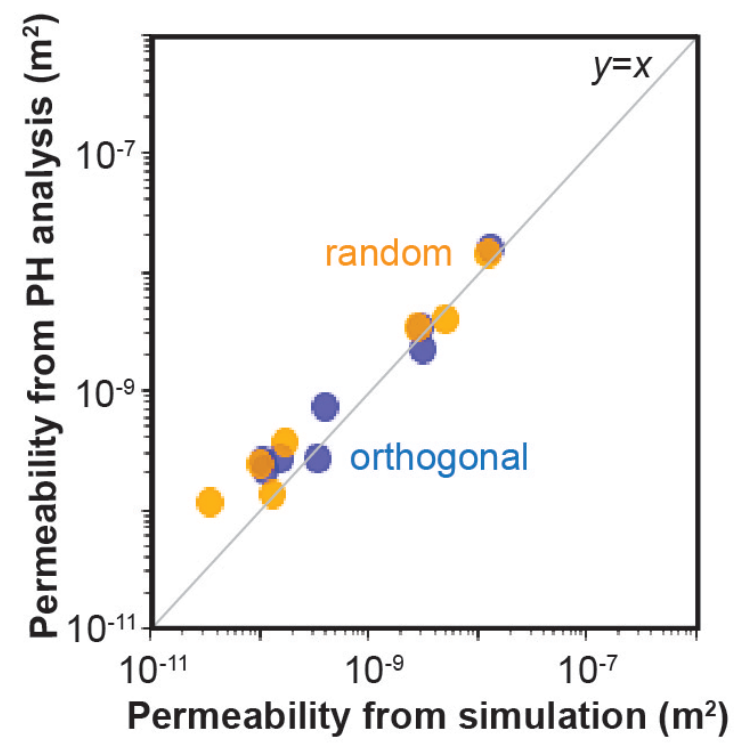

Figure 6 | Estimation of permeability by persistent homology (PH) analysis for orthogonal fracture networks (blue) and random fracture networks (orange). The calculated permeability is listed in Table 1.

There is a limitation of Eq. (7). Eq. (7) is based on a parallel plate model, so the flow is assumed to be straight. If there is tortuosity in a flow channel, the flow length will be longer, and the estimated permeability may be larger than the true value. Figure 7 shows streamlines in model $\mathrm{O} 8$ colored as green. We can see that the streamlines are winding and flowing. Keep in mind the fact that tortuosity was not taken into account in Eq. (7).
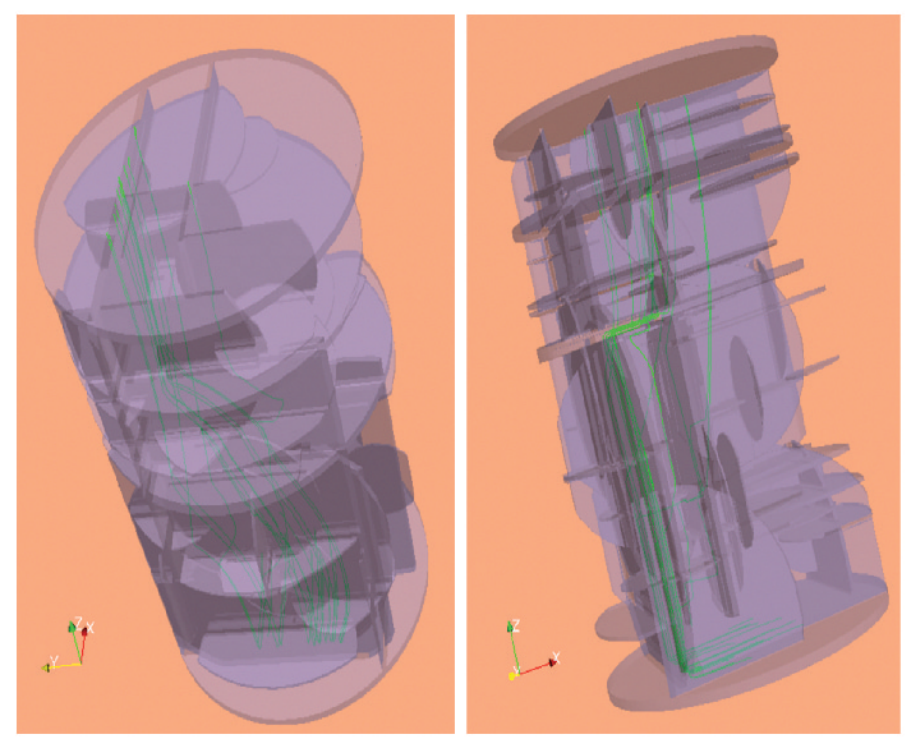

Figure 7 | Streamlines (green lines) in fracture network simulated in OpenFOAM.

We also applied persistent homology analysis to other cases. Mehmani and Mamdi $(2015)^{39}$ conducted high-fidelity direct numerical simulation of the two-dimensional micromodel to develop their pore network models. We used their 2D image data as shown in Figure $8 \mathrm{a}$ and their results from direct numerical simulation. Comparison with persistent 
Berea sandstone in Figure 8c. These results suggest that the proposed analysis can be used for two-dimensional flow.

Andrew et al. (2014) ${ }^{40}$ used X-ray microtomography to obtain four types of 3D rock image data, and they conducted flow experiment to measure the permeability. The X-ray microtomography images of the rocks are shown in Figure 8b. Comparison with the experimental results is plotted as green dots in Figure 8c. Permeability estimated by persistent homology is larger than the experimental results. As mentioned before, Eq. (7) does not consider the effect of tortuosity. Muljadi et al. (2016) ${ }^{41}$ calculated the tortuosity from the same Bentheimer sandstone and the Estaillades carbonate images as 1.52 and 1.91, respectively. If we take the tortuosity into account, the estimates of the permeability will be close to the experimental values. The calculation of tortuosity in Muljadi et al. (2016) ${ }^{41}$ used the flow velocity ${ }^{42,43}$. In contrast, the goal of this study is to estimate flow properties without flow simulation, so that we need to obtain tortuosity in a different way based on image analysis. Correlation between tortuosity and persistent homology parameters would be explored in future studies.
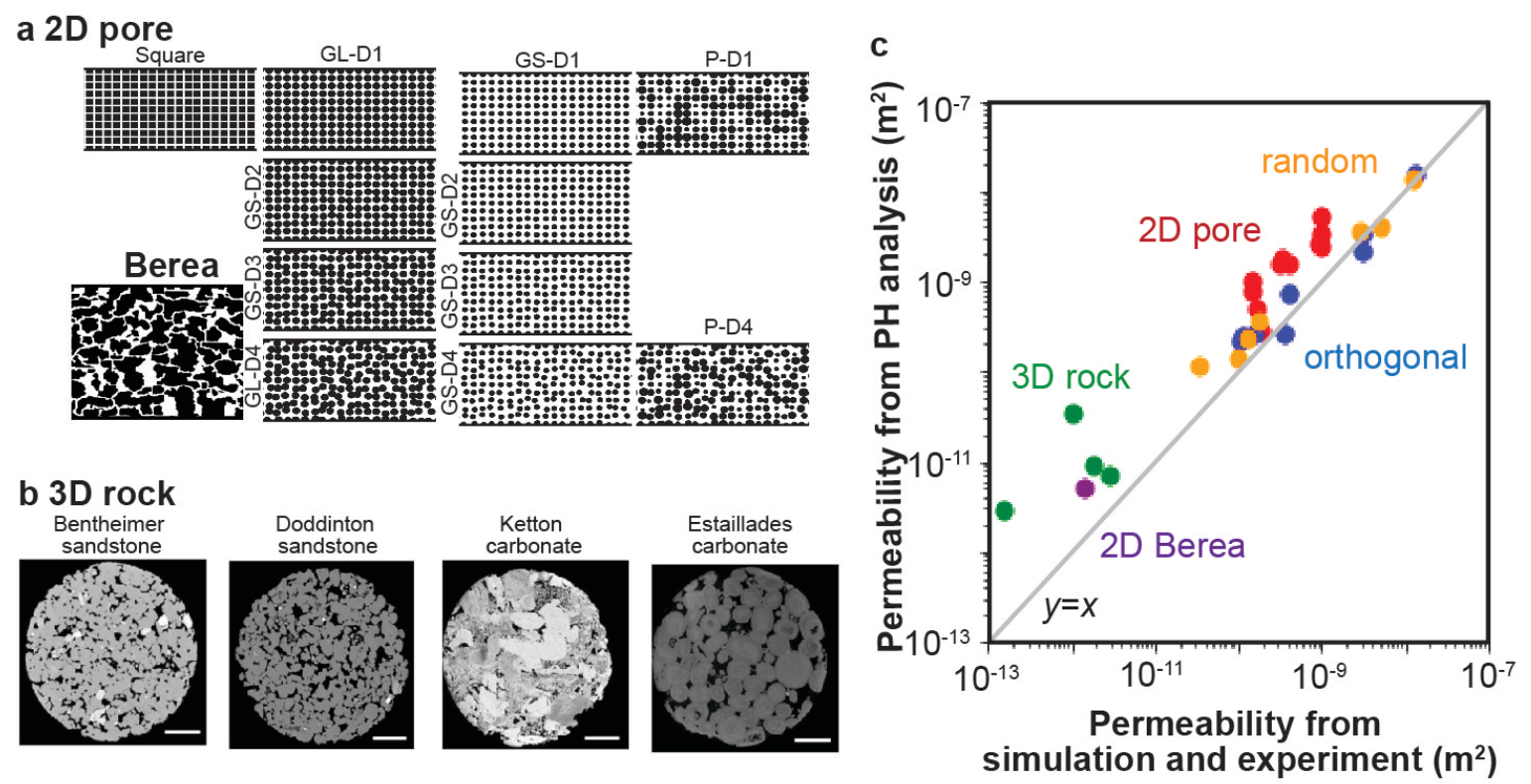

Figure 8 | Estimation of permeability by persistent homology $(\mathrm{PH})$ analysis. a $2 \mathrm{D}$ images from Mehmani and Hamdi $(2015)^{39}$, b 3D rock images from Andrew et al. $(2014)^{40}$, and c comparison with direct simulation and experiment. The values are listed in Table 3. 
Table 3 | Information of 2D pore ${ }^{39}$ and 3D rock images ${ }^{40}$ and their permeability.

\begin{tabular}{|c|c|c|c|c|}
\hline Model & $\begin{array}{c}\text { Image } \\
\text { size } \\
\text { (pixels) }\end{array}$ & $\begin{array}{c}\text { Domain } \\
\text { size } \\
(\mathrm{mm})\end{array}$ & $\begin{array}{l}\text { Simulation } \\
\text { result }\end{array}$ & $\begin{array}{c}\mathrm{PH} \\
\text { estimation }\end{array}$ \\
\hline \multicolumn{5}{|l|}{ 2D pore } \\
\hline Square & \multirow{11}{*}{$\begin{array}{c}3000 x \\
1500\end{array}$} & \multirow{11}{*}{$\begin{array}{l}20 \mathrm{~mm} \\
\times 10 \mathrm{~mm} \\
\times 200 \mu \mathrm{m}\end{array}$} & $3.13 \times 10^{-10}$ & $1.54 \times 10^{-9}$ \\
\hline GL-D1 & & & $1.92 \times 10^{-10}$ & $2.74 \times 10^{-10}$ \\
\hline GL-D2 & & & $1.70 \times 10^{-10}$ & $4.96 \times 10^{-10}$ \\
\hline GL-D3 & & & $1.47 \times 10^{-10}$ & $7.98 \times 10^{-10}$ \\
\hline GL-D4 & & & $1.44 \times 10^{-10}$ & $9.71 \times 10^{-10}$ \\
\hline GS-D1 & & & $9.77 \times 10^{-10}$ & $5.19 \times 10^{-9}$ \\
\hline GS-D2 & & & $9.75 \times 10^{-10}$ & $3.34 \times 10^{-9}$ \\
\hline GS-D3 & & & $9.60 \times 10^{-10}$ & $2.43 \times 10^{-9}$ \\
\hline GS-D4 & & & $9.18 \times 10^{-10}$ & $2.67 \times 10^{-9}$ \\
\hline P-D1 & & & $3.25 \times 10^{-10}$ & $1.75 \times 10^{-9}$ \\
\hline$P-D 4$ & & & $4.01 \times 10^{-10}$ & $1.53 \times 10^{-9}$ \\
\hline Berea & $\begin{array}{c}2900 x \\
2320\end{array}$ & $\begin{array}{r}1.774 \mathrm{~mm} \\
\times 1.418 \mathrm{~mm} \\
\times 24.54 \mu \mathrm{m} \\
\end{array}$ & $1.45 \times 10^{-12}$ & $5.06 \times 10^{-12}$ \\
\hline Model & $\begin{array}{c}\text { Image } \\
\text { size } \\
\text { (voxels) }\end{array}$ & $\begin{array}{l}\text { Resolution } \\
\text { (um/px) }\end{array}$ & $\begin{array}{l}\text { Experimental } \\
\text { result }\end{array}$ & $\begin{array}{c}\mathrm{PH} \\
\text { estimation }\end{array}$ \\
\hline \multicolumn{5}{|l|}{ 3D rock } \\
\hline Doddington & & 2.6929 & $1.04 \times 10^{-12}$ & $3.37 \times 10^{-11}$ \\
\hline Bentheimer & $300 \times 300$ & 3.0035 & $1.88 \times 10^{-12}$ & $9.02 \times 10^{-11}$ \\
\hline Ketton & x 300 & 3.00006 & $2.81 \times 10^{-12}$ & $6.86 \times 10^{-11}$ \\
\hline Estaillades & & 3.31136 & $1.49 \times 10^{-13}$ & $2.90 \times 10^{-11}$ \\
\hline
\end{tabular}

\section{Discussions}

Persistent homology analysis could estimate opening aperture distributions of flow channels and estimated permeability with the same order of magnitude as the permeability derived from the simulation. Using this method, flow characteristics can be estimated from the image data without the need for fluid flow simulation. This could make the analysis of fracture networks quicker. In this study, the longest direct flow simulation took 72 hours to generate a sufficiently high-resolution computational mesh then solve the Navier-Stokes equations, using 320 processors with a maximum of $282 \mathrm{~GB}$ of memory in a supercomputational system. In contrast, persistent homology was able to calculate the model in less than 10 minutes with 16 GB of memory using a desktop workstation AMD Ryzen 9 5950X.

Several approaches ${ }^{44-46}$ based on discrete fracture network represent fractures as ellipses or rectangles in networks based on Eq. (2). Focusing on the fractures themselves is suitable for fractured rock bodies, but it may be difficult to optimize the model because of the increase in number of parameters when the fractures are finer or when the body is regarded as a porous medium. In this study, we focus on the flow channels by persistent homology instead of individual fractures. Therefore, we can apply the method regardless of porous or fractured rocks.

Recently, some studies have been published to investigate the relationship between porous structures and flow by persistent homology 29,30,47,48. Most of them were machine learning approaches that put a large number of parameters into a black box. In contrast, since our approach focuses on flow-channel structures, permeability can be calculated by the simple and easy principle. We used the synthetic fracture networks as well as natural rocks. Although the estimation errors were relatively large for 3D rocks, it was shown that a simple model such as Eq. (7) can provide reasonably close estimates.

Ushizima et al. (2012) ${ }^{26}$ estimates permeability of porous rocks by using Reeb graphs to represent the pore networks. They use persistent homology to distinguish between significant and noisy pore spaces, and to supplement the Reeb graphs. In fact, the Reeb graph 
and persistent homology were used independently and separately. We think that using Reeb graphs is a good direction to go to the next step.

We have succeeded in modeling physical phenomena from image data based on the topological data analysis. The method could be applied also to a wide range of porous media including artificial devices. It is expected to be applicable not only to estimate flow properties, but also to characterize different transport phenomena, such as mass transfer, electrical and magnetic flows.

\section{Method \\ Persistent homology analysis}

STL files of synthetic fracture networks were generated by using OpenSCAD, and the STL files were converted to the cross-sectional images in $36 \mathrm{~mm} \times 36 \mathrm{~mm} \times 50 \mathrm{~mm}$ with a voxel resolution of $0.1 \mathrm{~mm}$ in Autodesk Netfabb. To eliminate some unexpected noises, all the images were blurred in XnConvert. The png files of the image data were analyzed in HomCloud $^{63}$. When there were small differences between birth and death of $\mathrm{PH}_{1}$, the hole structure may appear during the image analysis. Thus, we neglected the result with $d_{i}-b_{i}<2$ were eliminated.

\section{Direct flow simulation}

Flow behaviors in the rock models were simulated in OpenFOAM (ver. 4.1) ${ }^{34}$, which performs flow calculations based on the Navier-Stokes equation. The simulation used an STL file of the network model, in which the upper and lower boundaries were added, as shown in Figure 9a. Previous study validated the flow model with fracture networks similar to the rock model in this study by creating test specimens from a 3D printer and conducting flow experiment ${ }^{49,50}$.

First, the meshes of the region were prepared (Figure 9b), and flow calculation of the loaded STL file were performed using a steady-state turbulence solver for incompressible fluids SIMPLE (Semi-Implicit Method for Pressure Linked Equations) method. In this study, we set the flow rate to $1.75 \times 10^{-7} \mathrm{~m}^{3} / \mathrm{s}$, fluid viscosity to $9.32 \times 10^{-4} \mathrm{~Pa} \cdot \mathrm{s}$, and fluid density to $997.5 \mathrm{~kg} / \mathrm{m}^{3}$.
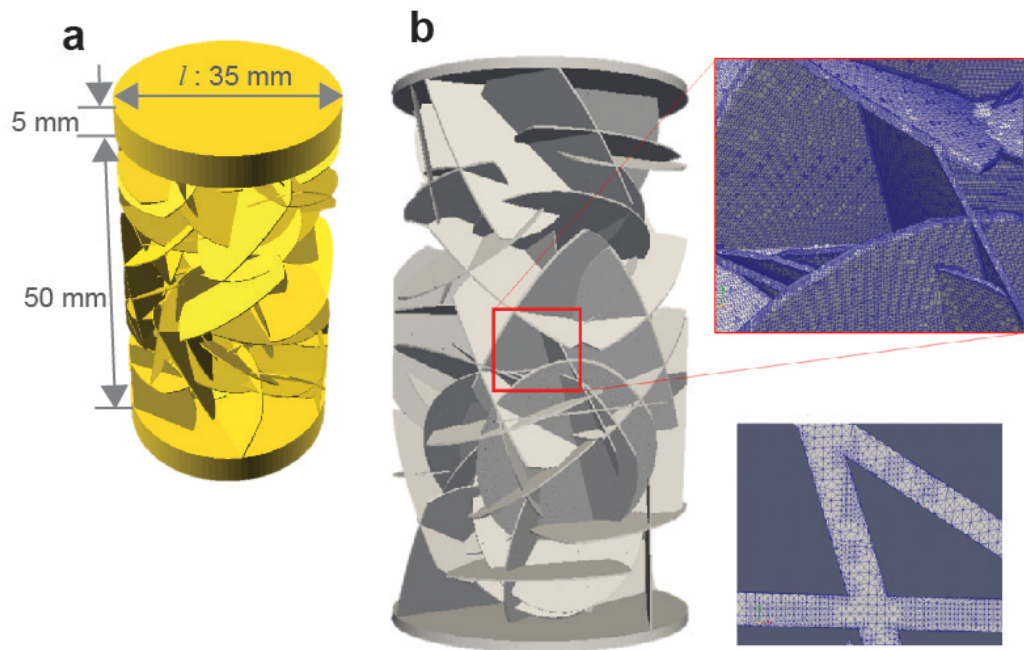

Figure 9 | Simulation in OpenFOAM. a fracture network with upper and lower boundaries. $\mathbf{b}$ discretized model.

\section{Data availability}


The data that support the findings of this study are available in

https://figshare.com/s/cebf663b253d145bf8ac (doi: 10.6084/m9.figshare.14110262, active

when the item is published), https://figshare.com/s/de6df5d1 cf76926c7f25 (doi:

10.6084/m9.figshare.14110208), https://figshare.com/s/96f9d83791dc03e28ce0 (doi:

\section{Reference}

438

439

440

1. Renard, P. \& de Marsily, G. Calculating equivalent permeability: a review. Adv. Water Resour. 20, 253-278 (1997).

2. Costa, A. Permeability-porosity relationship: A reexamination of the KozenyCarman equation based on a fractal pore-space geometry assumption. Geophys. Res. Lett. 33, 1-5 (2006).

3. Carman, P. C. Fluid flow through granular beds. Trans. Chem. Eng. 15, S32S48 (1937).

4. Zimmerman, R. W. \& Bodvarsson, G. S. Effective transmissivity of twodimensional fracture networks. Int. J. Rock Mech. Min. Sci. Geomech. Abstr. 33, 433-438 (1996).

5. Snow, D. Anisotropic Permeability o[ Fractured Media. Water Resour. Res. 5, 1273-1289 (1969).

6. Renshaw, C. E. On the relationship between mechanical and hydraulic apertures in rough-walled fractures. J. Geophys. Res. 100, 629-636 (1995).

7. Witherspoon, P. A., Wang, J. S. Y., Iwai, K. \& Gale, J. E. Validity of Cubic Law for fluid flow in a deformable rock fracture. Water Resour. Res. 16, 1016-1024 (1980).

8. Zimmerman, R. \& Yeo, I. Fluid Flow in Rock Fractures: From the NavierStokes Equations to the Cubic Law. Dyn. fluids Fract. rock 213-224 (2000). doi:10.1029/GM122p0213

9. Hyman, J. D., Aldrich, G., Viswanathan, H., Makedonska, N. \& Karra, S. Fracture size and transmissivity correlations: Implications for transport simulations in sparse three-dimensional discrete fracture networks following a truncated power law distribution of fracture size. Water Resour. Res. 5, 2-2 (1969).

10. Valentini, L., Perugini, D. \& Poli, G. The "small-world" topology of rock fracture networks. Phys. A Stat. Mech. its Appl. 377, 323-328 (2007).

11. Andresen, C. A., Hansen, A., Le Goc, R., Davy, P. \& Hope, S. M. Topology of fracture networks. Front. Phys. 1, 1-5 (2013).

12. Mecke, K. \& Arns, C. H. Fluids in porous media: A morphometric approach. J. Phys. Condens. Matter 17, (2005).

13. Lehmann, P. et al. Impact of geometrical properties on permeability and fluid phase distribution in porous media. Adv. Water Resour. 31, 1188-1204 (2008).

14. Scholz, C. et al. Permeability of porous materials determined from the Euler characteristic. Phys. Rev. Lett. 109, 1-5 (2012).

15. Liu, Z., Herring, A., Arns, C., Berg, S. \& Armstrong, R. T. Pore-Scale Characterization of Two-Phase Flow Using Integral Geometry. Transp. Porous Media 118, 99-117 (2017).

16. Armstrong, R. T. et al. Porous Media Characterization Using Minkowski Functionals: Theories, Applications and Future Directions. Transp. Porous Media 130, 305-335 (2019).

17. Edelsbrunner, H. \& Harer, J. Persistent homology-a survey. Contemp. Math. 257-282 (2008). doi:10.1090/conm/453/08802 
18. Zomorodian, A. \& Carlsson, G. Computing persistent homology. Proc. Annu. Symp. Comput. Geom. 274, 347-356 (2004).

19. Edelsbrunner, H. \& Morozov, D. Persistent Homology : Theory and Practice. in Conference: European Congress of Mathematics (2012).

20. Weinberger, S. What is Persistent Homology? Am. Math. Soc. 58, 36-39 (2010).

21. Chazal, F. \& Michel, B. An introduction to topological data analysis: Fundamental and practical aspects for data scientists. arXiv 1-38 (2017).

22. Otter, N., Porter, M. A., Tillmann, U., Grindrod, P. \& Harrington, H. A. A roadmap for the computation of persistent homology. EPJ Data Sci. 6, (2017).

23. Kimura, M., Obayashi, I., Takeichi, Y., Murao, R. \& Hiraoka, Y. Non-empirical identification of trigger sites in heterogeneous processes using persistent homology. Sci. Rep. 8, 1-9 (2018).

24. Hiraoka, Y. et al. Hierarchical structures of amorphous solids characterized by persistent homology. Proc. Natl. Acad. Sci. U. S. A. 113, 7035-7040 (2016).

25. Ichinomiya, T., Obayashi, I. \& Hiraoka, Y. Persistent homology analysis of craze formation. Phys. Rev. E 95, 1-6 (2017).

26. Ushizima, D. et al. Augmented topological descriptors of pore networks for material science. IEEE Trans. Vis. Comput. Graph. 18, 2041-2050 (2012).

27. Robins, V., Saadatfar, M., Delgado-Friedrichs, O. \& Sheppard, A. P. Percolating length scales from topological persistence analysis of micro-CT images of porous materials. Water Resour. Res. 52, 315-329 (2016).

28. Tsuji, T., Jiang, F., Suzuki, A. \& Shirai, T. Mathematical Modeling of Rock Pore Geometry and Mineralization: Applications of Persistent Homology and Random Walk. 95-109 (2018). doi:10.1007/978-981-10-7811-8_11

29. Jiang, F., Tsuji, T. \& Shirai, T. Pore Geometry Characterization by Persistent Homology Theory. Water Resour. Res. 54, 4150-4163 (2018).

30. Herring, A. L., Robins, V. \& Sheppard, A. P. Topological Persistence for Relating Microstructure and Capillary Fluid Trapping in Sandstones. Water Resour. Res. 55, 555-573 (2019).

31. Suzuki, A. et al. Inferring fracture forming processes by characterizing fracture network patterns with persistent homology. Comput. Geosci. 143, 104550 (2020).

32. Obayashi, I. \& Hiraoka, Y. Persistence diagrams with linear machine learning models. arXiv 1, 421-449 (2017).

33. Choudhury, a. N. M. I., Wang, B., Rosen, P. \& Pascucci, V. Topological analysis and visualization of cyclical behavior in memory reference traces. 2012 IEEE Pacific Vis. Symp. 9-16 (2012). doi:10.1109/PacificVis.2012.6183557

34. Choudhury, A. N. M. I., Wang, B., Rosen, P. \& Pascucci, V. Topological analysis and visualization of cyclical behavior in memory reference traces. IEEE Pacific Vis. Symp. 2012, PacificVis 2012 - Proc. 9-16 (2012). doi:10.1109/PacificVis.2012.6183557

35. Martys, N. \& Garboczi, E. J. Length scales relating the quid permeability and electrical conductivity in random two-dimensional model porous media. Phys. Rev. B 46, 6080-6090 (1992).

36. Schwartz, L. M., Martys, N., Bentz, D. P., Garboczi, E. J. \& Torquato, S. Cross-property relations and permeability estimation in model porous media. Phys. Rev. E 48, 4584-4591 (1993).

37. Nishiyama, N. \& Yokoyama, T. Permeability of porous media: Role of the 
critical pore size. J. Geophys. Res. Solid Earth 122, 6955-6971 (2017).

38. Watanabe, K. \& Takahashi, H. Fractal geometry characterization of geothermal reservoir fracture networks. Journal of Geophysical Research 100, 521-528 (1995).

39. Mehmani, Y. \& Tchelepi, H. A. Minimum requirements for predictive porenetwork modeling of solute transport in micromodels. Adv. Water Resour. 108, 83-98 (2017).

40. Andrew, M., Bijeljic, B. \& Blunt, M. J. Pore-scale imaging of trapped supercritical carbon dioxide in sandstones and carbonates. Int. J. Greenh. Gas Control 22, 1-14 (2014).

41. Muljadi, B. P., Blunt, M. J., Raeini, A. Q. \& Bijeljic, B. The impact of porous media heterogeneity on non-Darcy flow behaviour from pore-scale simulation. Adv. Water Resour. 95, 329-340 (2016).

42. Duda, A., Koza, Z. \& Matyka, M. Hydraulic tortuosity in arbitrary porous media flow. Phys. Rev. E - Stat. Nonlinear, Soft Matter Phys. 84, 1-8 (2011).

43. Koponen, I. Analytic approach to the problem of convergence of truncated Levy flights towards the Gaussian stochastic process. Phys. Rev. E 52, 11971199 (1995).

44. Jing, Y., Armstrong, R. T. \& Mostaghimi, P. Image-based fracture pipe network modelling for prediction of coal permeability. Fuel 270, 117447 (2020).

45. Srinivasan, S., Karra, S., Hyman, J., Viswanathan, H. \& Srinivasan, G. Model reduction for fractured porous media: a machine learning approach for identifying main flow pathways. Comput. Geosci. 23, 617-629 (2019).

46. Srinivasan, G. et al. Quantifying Topological Uncertainty in Fractured Systems using Graph Theory and Machine Learning. Sci. Rep. 8, 1-11 (2018).

47. Robinson, J. et al. Imaging pathways in fractured rock using three-dimensional electrical resistivity tomography. Groundwater 54, 186-201 (2016).

48. Thakur, M. M., Kim, F., Penumadu, D. \& Herring, A. Pore Space and Fluid Phase Characterization in Round and Angular Partially Saturated Sands Using Radiation-Based Tomography and Persistent Homology. Transp. Porous Media (2021). doi:10.1007/s11242-021-01554-w

49. Suzuki, A., Watanabe, N., Li, K. \& Horne, R. N. Fracture network created by 3D printer and its validation using CT images. Water Resour. Res. (2017). doi:10.1002/2017WR021032

50. Suzuki, A., Minto, J. M., Watanabe, N., Li, K. \& Horne, R. N. Contributions of 3D Printed Fracture Networks to Development of Flow and Transport Models. Transp. Porous Media 129, 485-500 (2019).

\section{Acknowledgements}

Anna Suzuki was supported by JSPS KAKENHI Grant Numbers JP20H02676 and JP17H04976 (Japan); JST ACT-X Grant Number JPMJAX190H (Japan). Ippei Obayashi was supported by JSPS KAKENHI Grant Number JP 16K17638, JP 19H00834, JP 20H05884; JST PRESTO Grant Number JPMJPR1923; JST CREST Mathematics Grant number 15656429 (Japan); and the Structural Materials for Innovation, Strategic Innovation Promotion Program D72 (Japan), which are gratefully acknowledged. The authors would like to thank Department of Earth Science and Engineering, Imperial College London for sharing the micro-CT data of the rocks. These micro-CT data can be downloaded through their web page: http://www.imperial.ac.uk/earth-sci- ence/research/research-groups/perm/ research/pore-scale-modelling/micro- ct-images-and-networks/. 
582 A.S. initiated the key concepts, designed, conducted persistent homology analysis, and 583 supervised the research. M.M. performed numerical simulation and conducted persistent 584 homology analysis. J.M. assisted flow simulation. I.O. and Y.H. assisted persistent homology 585 analysis. T.T and T.I conducted project administration. A.S. wrote the original draft. J.M., 586 I.O., Y.H., T.T and T.I reviewed and edited the manuscript.

\section{Conflicts of Interest}

589 The authors declare no conflict of interest. 


\section{Figures}

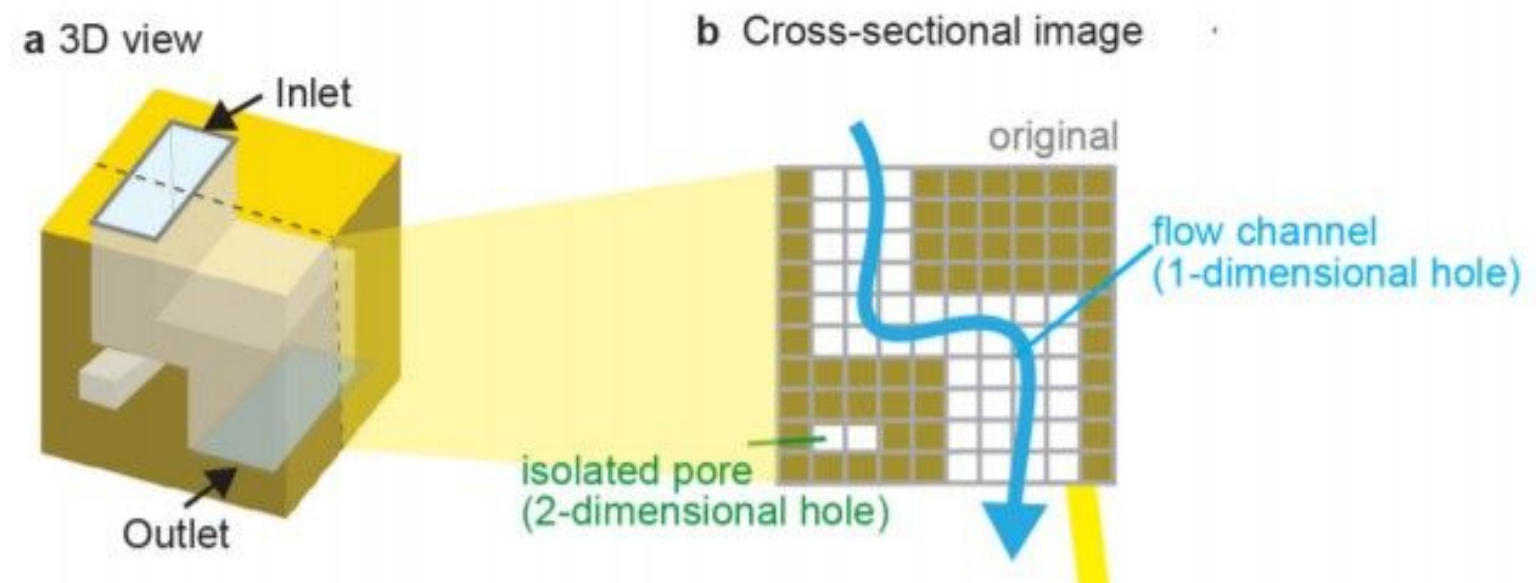

c Filtration

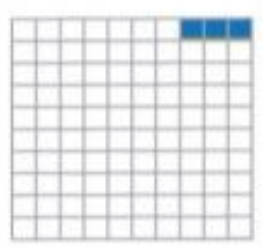

$t=-5$

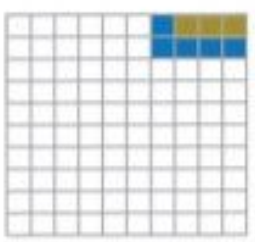

$t=-4$

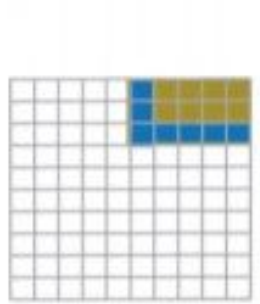

$t=-3$

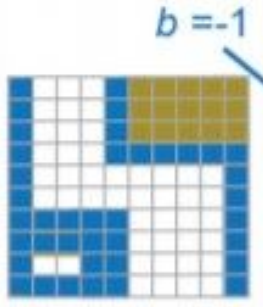

$t=-2$

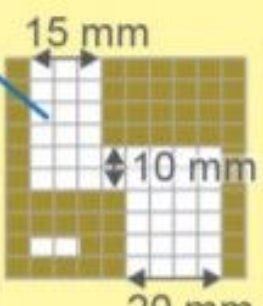

$t=-1$

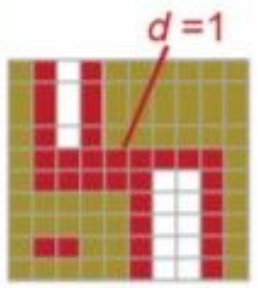

$t=1$

time

\section{Figure 1}

Persistent homology analysis for fracture networks by HomCloud32 . a 3D view and b cross-sectional image of fracture network with a flow channel (light blue arrow) and an isolated pore (mentioned as green). $c$ Schematic of filtration process. Image at $t=-1$ is the original image. White grids express void spaces. Blue grids are the grids that were removed during the thinning process. Red grids are the grids that were added during the thickening process. 


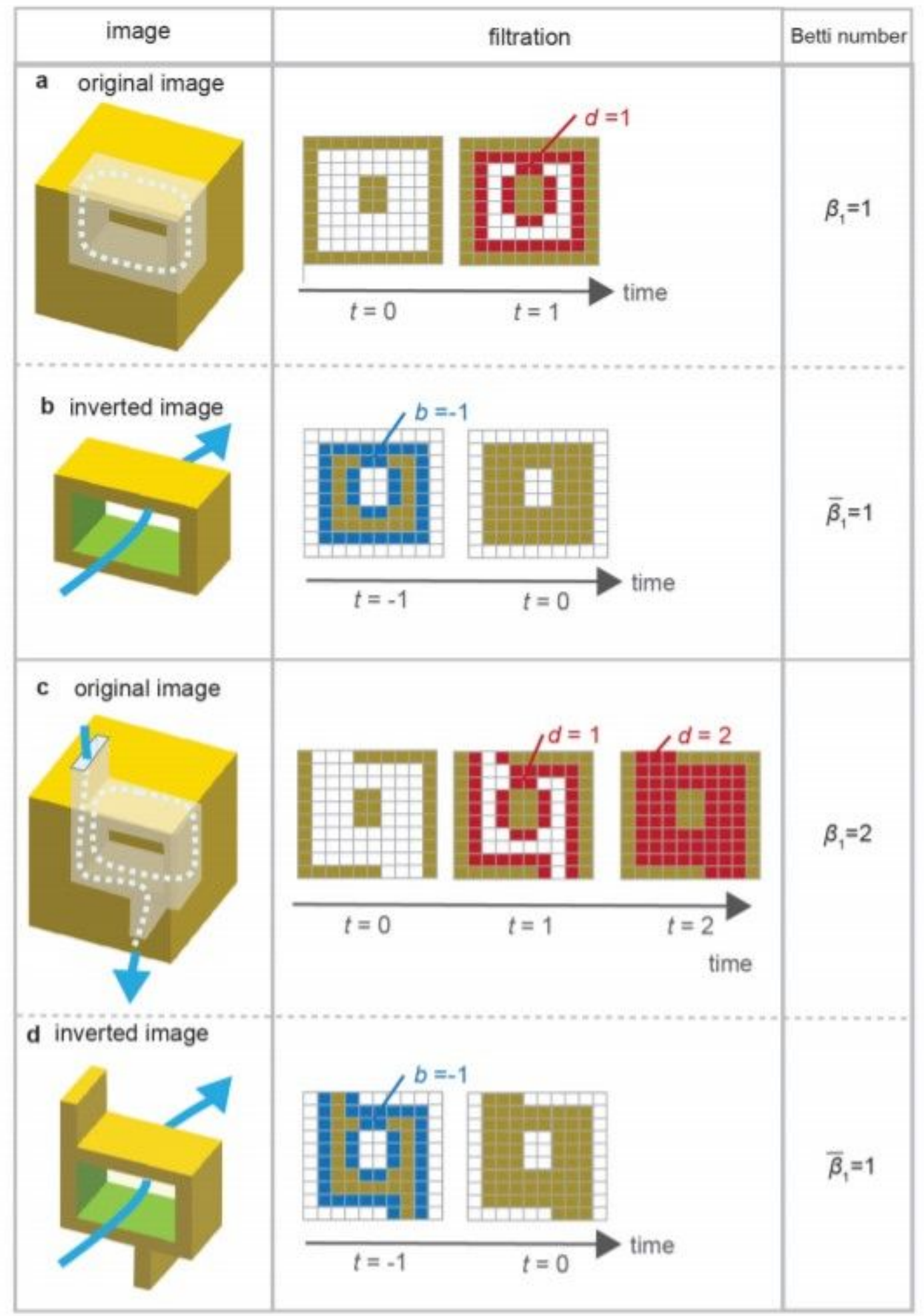

\section{Figure 2}

Detecting flow channels using inverted images a ring-shaped, internal void-structure that is not connected to the outside, and $b$ its inverted image. $c$ ring shaped, internal void-structure with two channels that is connected to the outside and forms a flow channel. $d$ its inverted image. The left column shows 3D view of images. The center column describes processes of filtration. The right column lists Betti numbers $\beta 1$. 
a

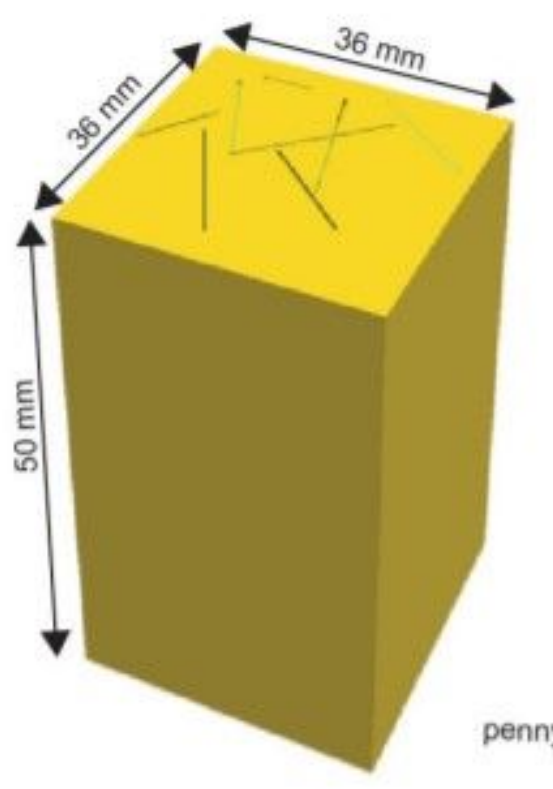

c Orthogonal distribution

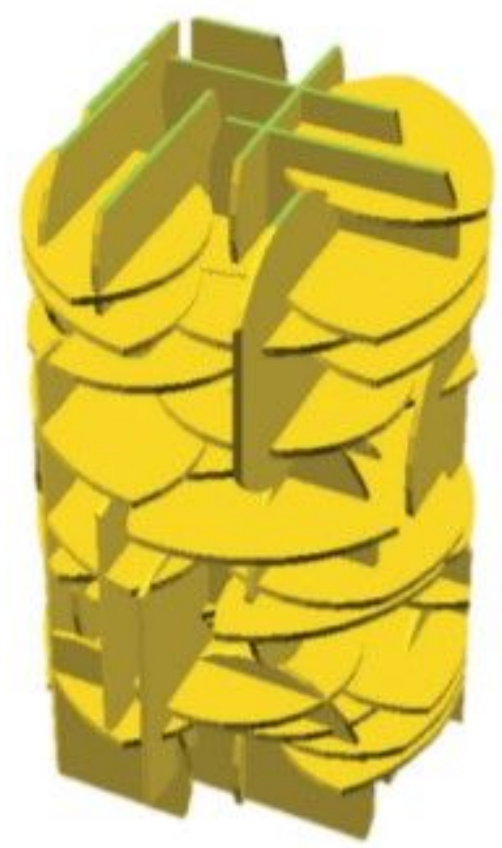

b

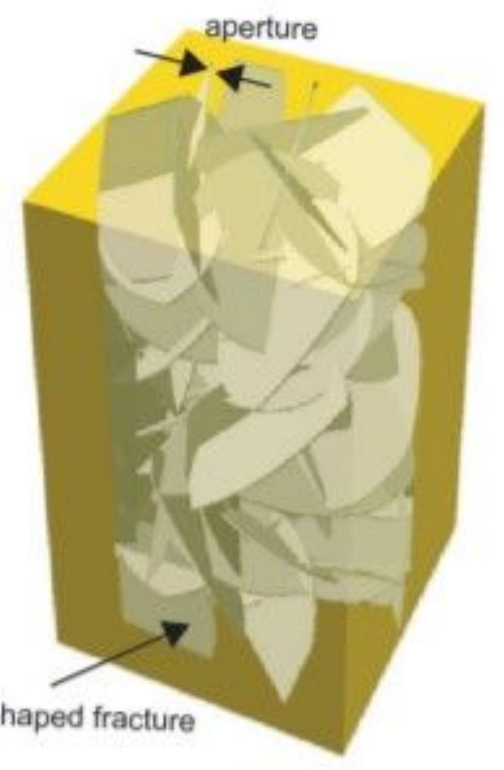

d Random distribution

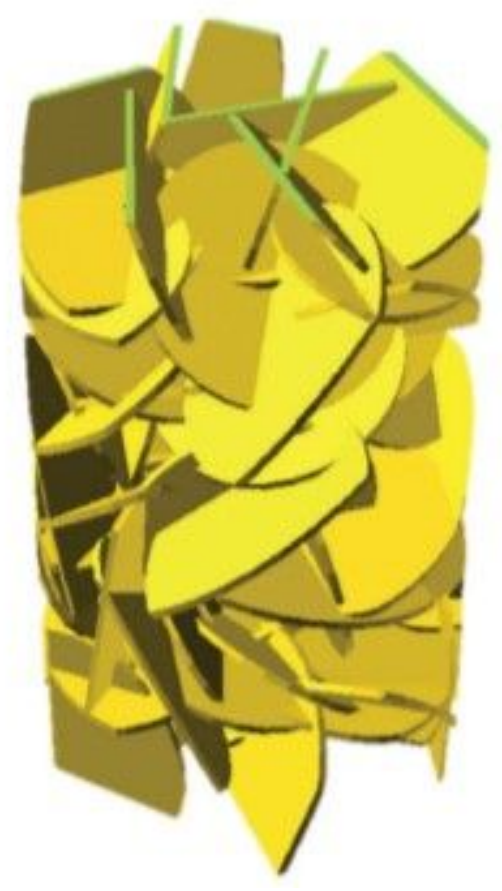

Figure 3

Fractured models. a outside and $\mathrm{b}$ inside of model. c Orthogonal distribution and $\mathrm{d}$ random distribution of fracture networks. 

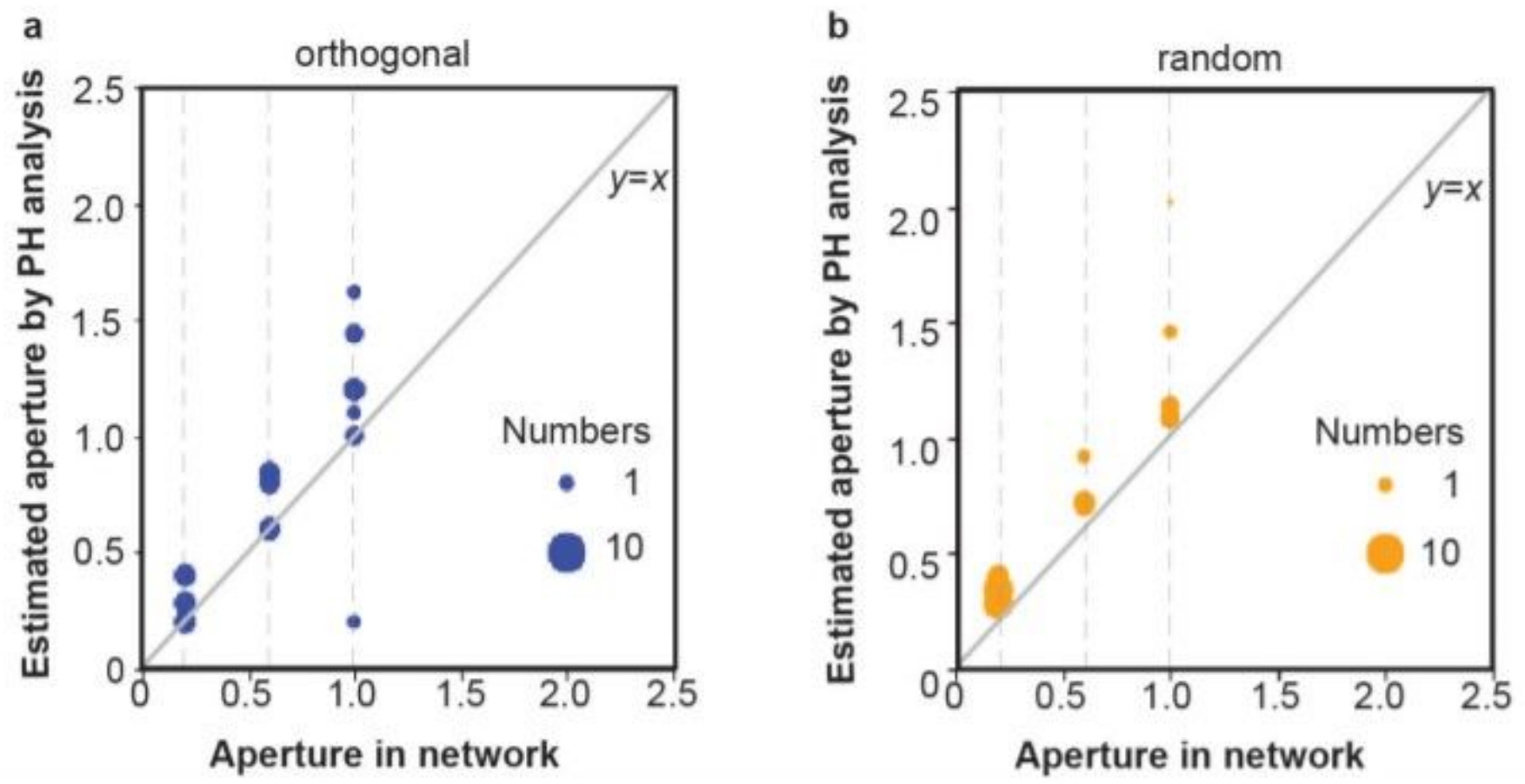

Figure 4

Estimation of fracture apertures by persistent homology $(\mathrm{PH})$ analysis. a orthogonal fracture networks and $b$ random fracture networks.

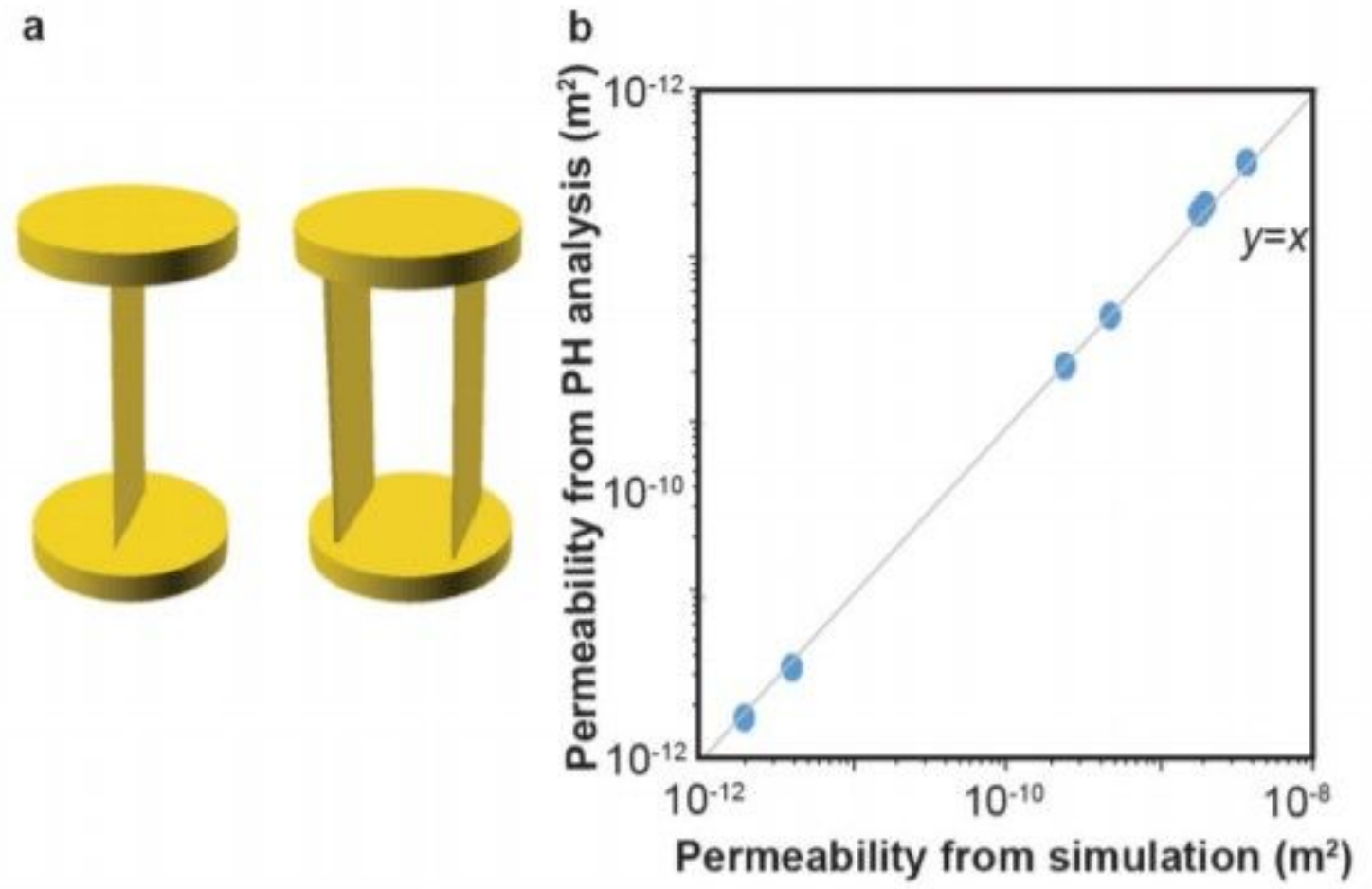

Figure 5

Validation with simple models. a one or two fracture penetrating the model. The top and bottom are the inlet and the outlet. b Comparison of permeability between persistent homology $(\mathrm{PH})$ analysis and direct 
simulation. The calculated permeability is listed in Table 2.

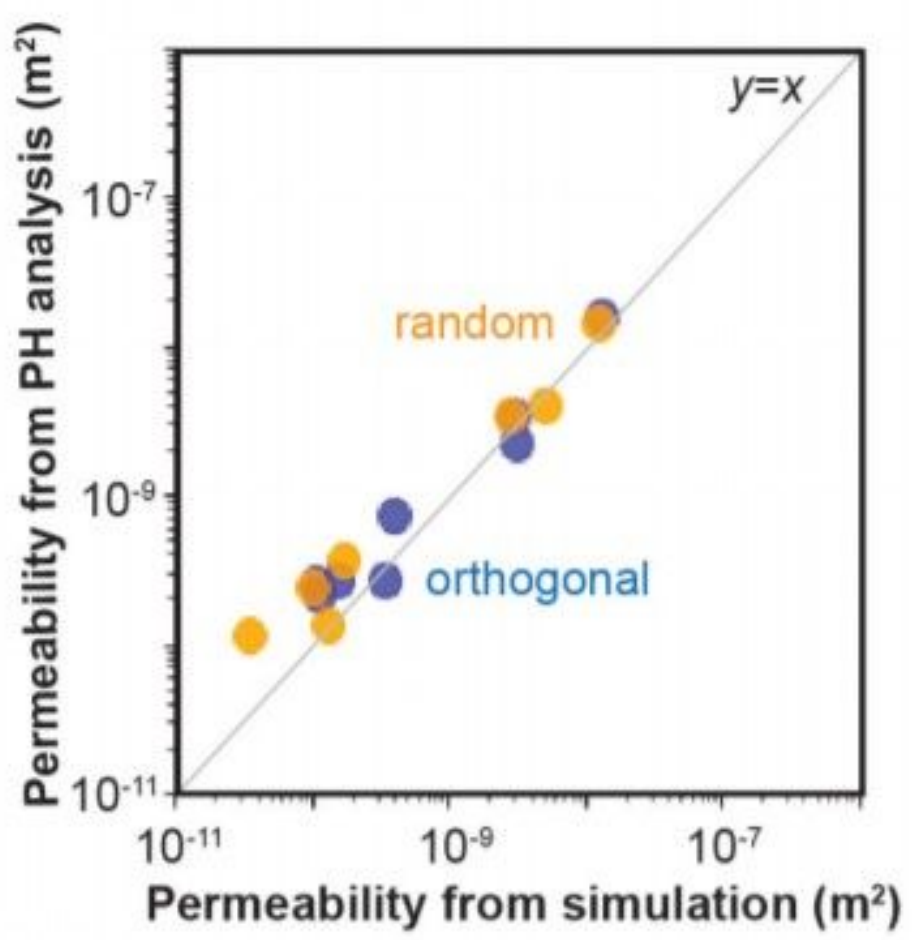

\section{Figure 6}

Estimation of permeability by persistent homology $(\mathrm{PH})$ analysis for orthogonal fracture networks (blue) and random fracture networks (orange). The calculated permeability is listed in Table 1.
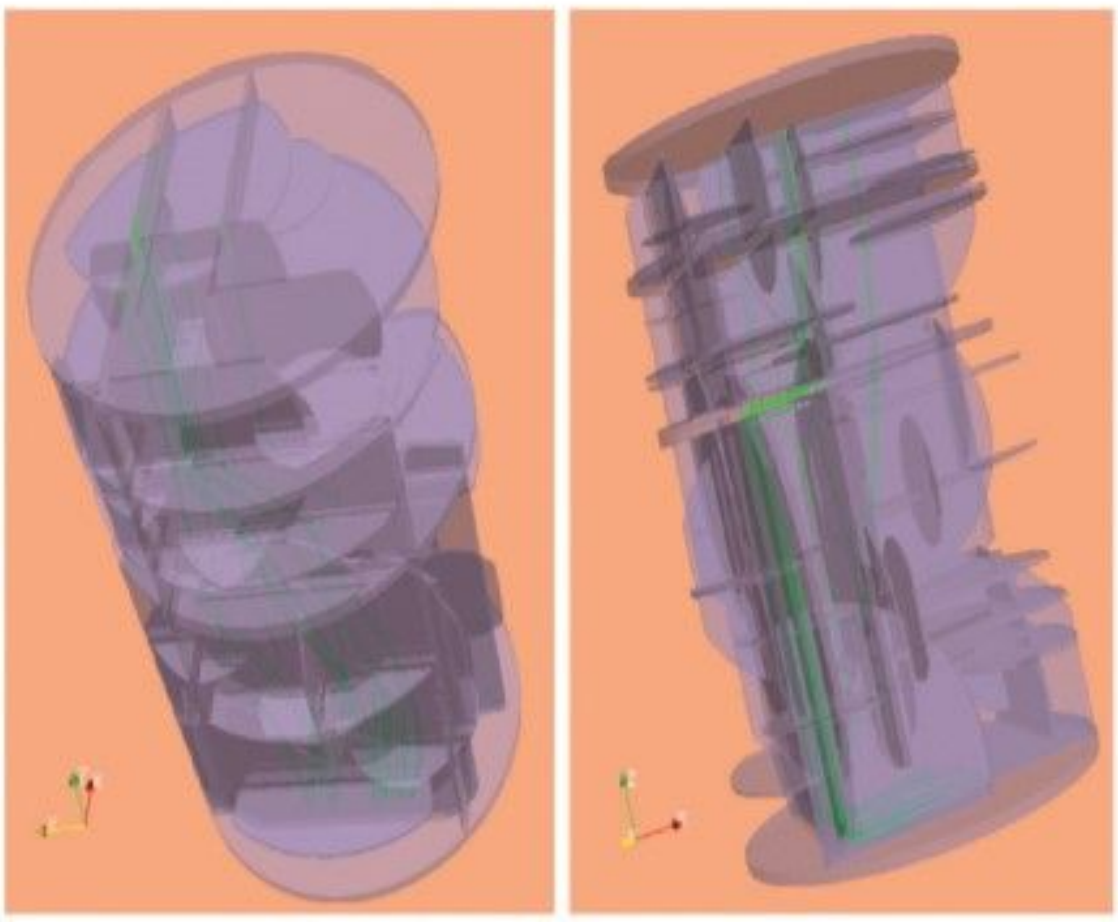

Figure 7 
Streamlines (green lines) in fracture network simulated in OpenFOAM
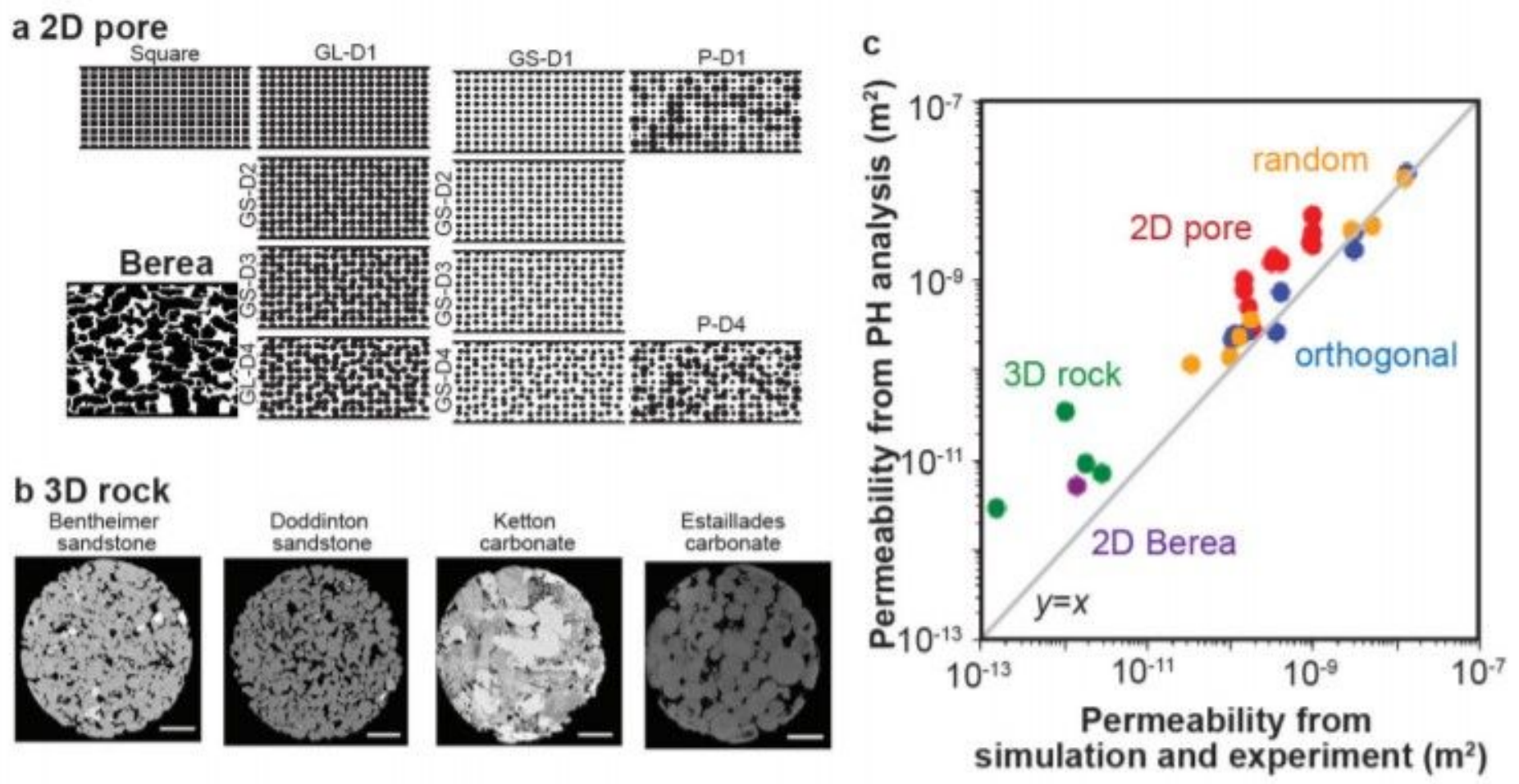

Figure 8

Estimation of permeability by persistent homology $(\mathrm{PH})$ analysis. a 2D images from Mehmani and Hamdi (2015)39, b 3D rock images from Andrew et al. (2014) 40 , and c comparison with direct simulation and experiment. The values are listed in Table 3.

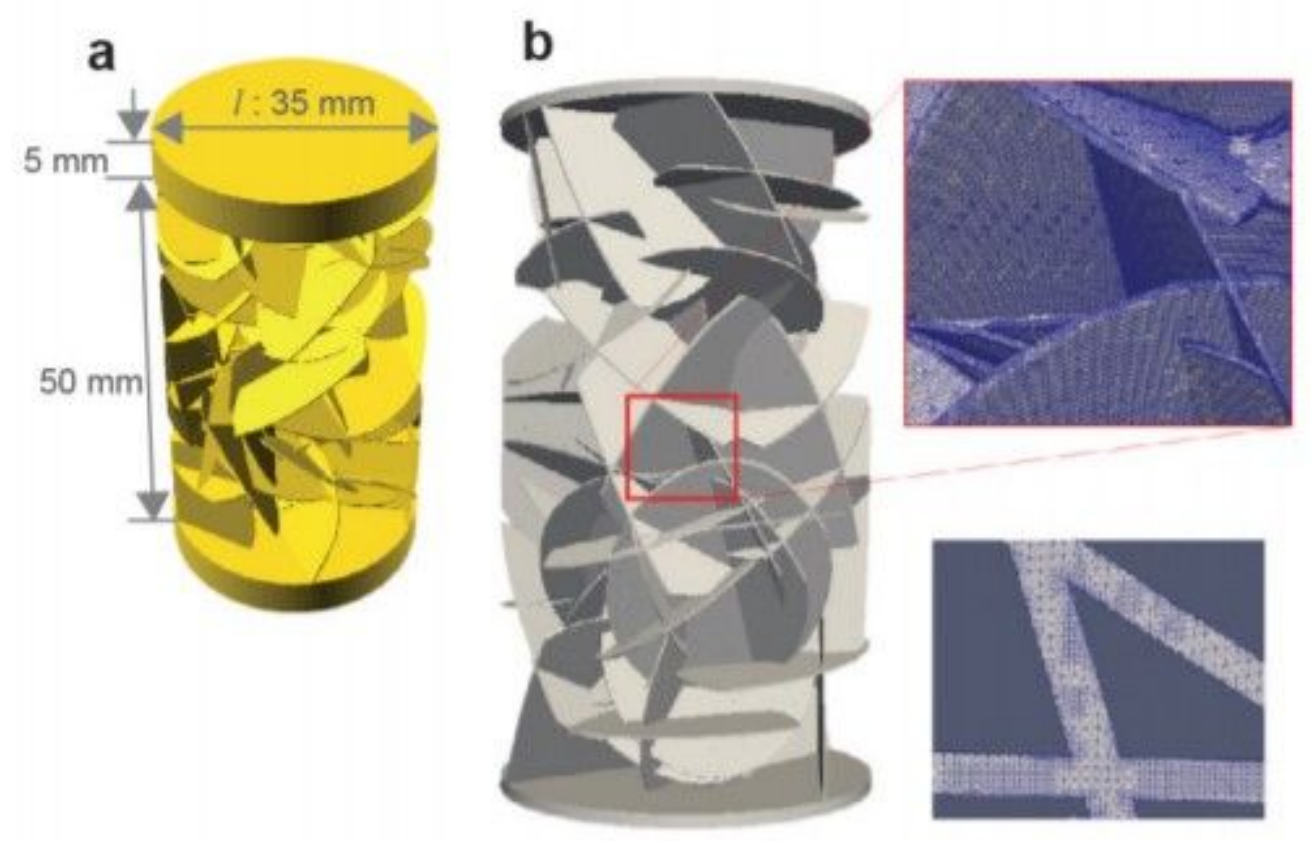

Figure 9 
Simulation in OpenFOAM. a fracture network with upper and lower boundaries. b discretized model. 